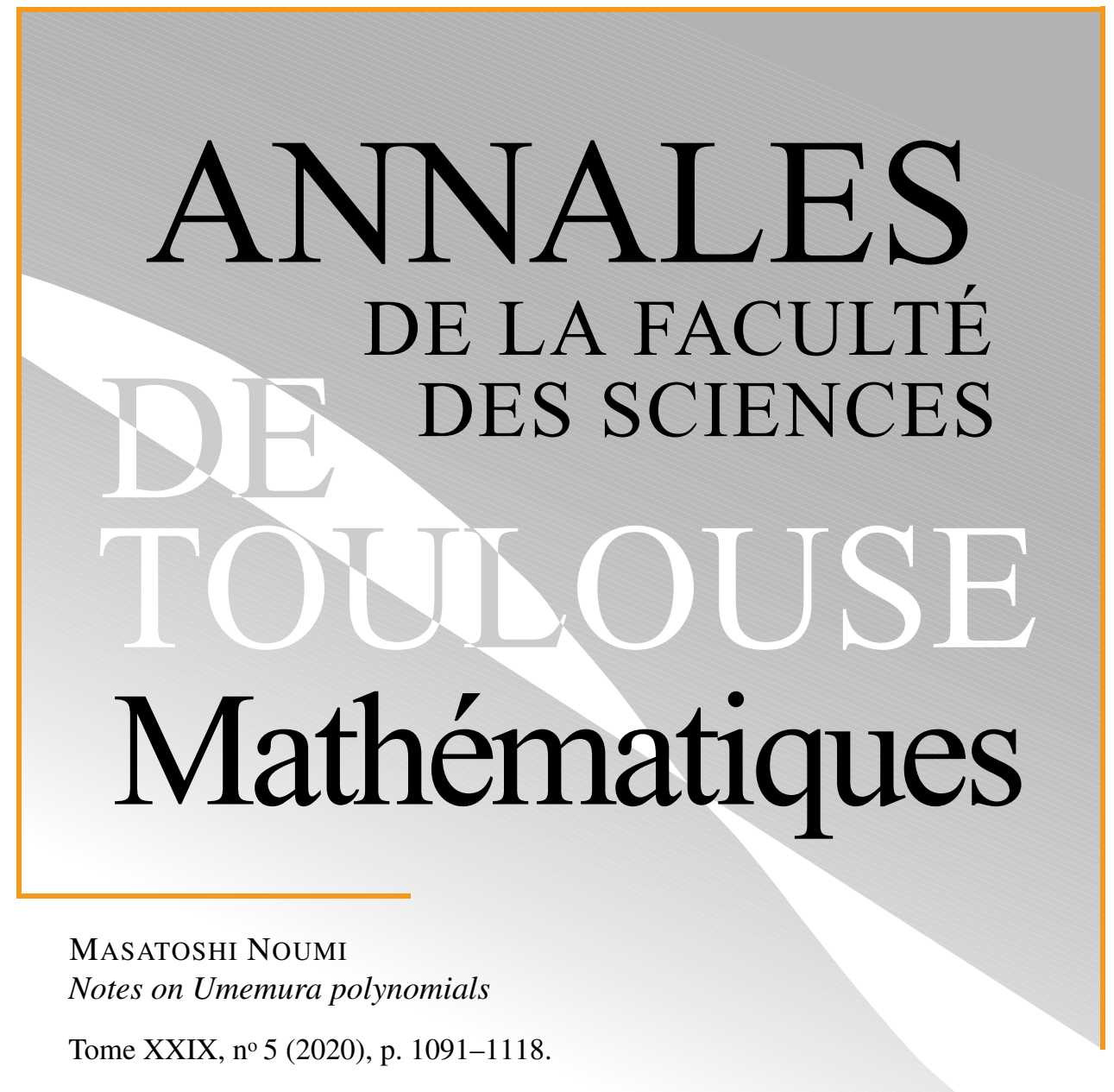

https://doi.org/10.5802/afst.1658

(C) Université Paul Sabatier, Toulouse, 2020.

L'accès aux articles de la revue «Annales de la faculté des sciences de Toulouse Mathématiques » (http://afst.centre-mersenne.org/) implique l'accord avec les conditions générales d'utilisation (http://afst.centre-mersenne.org/legal/). Les articles sont publiés sous la license CC-BY 4.0.

(c) $\underset{B \mathrm{BY}}{(1)}$

Publication membre du centre

Mersenne pour l'édition scientifique ouverte MERSENNE http://www.centre-mersenne.org/ 


\title{
Notes on Umemura polynomials
}

\author{
Masatoshi Noumi ${ }^{(1)}$
}

To the memory of Professor Hiroshi Umemura

\begin{abstract}
We give a survey on special polynomials associated with algebraic solutions of the sixth Painlevé equation, and formulate a conjecture regarding a combinatorial formula for Umemura polynomials associated with a class of algebraic solutions of $P_{\mathrm{VI}}$ with two discrete parameters.
\end{abstract}

RÉSumÉ. - Nous donnons un survol des polynômes spéciaux associés aux solutions algébriques de la sixième équation de Painlevé, et formulons une conjecture concernant une formule combinatoire pour les polynômes d'Umemura associés à une classe de solutions algébriques de $P_{\mathrm{VI}}$ avec deux paramètres discrets.

\section{Introduction}

The Umemura polynomials refer to the special polynomials associated with algebraic solutions of the sixth Painlevé equation $P_{\mathrm{VI}}$. In this paper we give a survey on special polynomials associated with algebraic solutions of the sixth Painlevé equation as discussed in [10], and formulate a conjecture regarding a combinatorial formula for Umemura polynomials associated with a class of algebraic solutions obtained by a two-parameter family of Bäcklund transformations as investigated by Masuda [8].

Consider the following recurrence formula of Toda type for the sequence $U_{n}=U_{n}(x)(n \in \mathbb{Z})$ of rational functions in $x$ :

$$
\begin{aligned}
U_{n-1} U_{n+1}=\left(x^{2}-1\right)^{2} & \left(x U_{n}^{\prime \prime} U_{n}+U_{n}^{\prime} U_{n}-x\left(U_{n}^{\prime}\right)^{2}\right) \\
& -\left(a^{2}(x+1)^{2}-b^{2}(x-1)^{2}-(2 n-1)^{2} x\right) U_{n}^{2},
\end{aligned}
$$

Keywords: Umemura polynomial, Painlevé equation, Toda equation, special polynomial.

2010 Mathematics Subject Classification: 33C45, 05E35, 34M55.

(1) Department of Mathematics, Kobe University, Rokko, Kobe 657-8501, Japan

This work was supported by JSPS Kakenhi Grant (B)15H03626. 
with initial condition $U_{0}=U_{1}=1$, where ${ }^{\prime}=\mathrm{d} / \mathrm{d} x$ stands for the derivation with respect to $x$, and $a, b$ are parameters. This recurrence formula originates from the Toda equation for a sequence of $\tau$ functions associated with a certain algebraic solution of the sixth Painlevé equation. Leaving aside the question how this recurrence formula was derived, we make here some comments on remarkable features of this recurrence formula.

With the initial data $U_{0}=U_{1}=1$, the second member is given by $U_{2}=a^{2}(x+1)^{2}-b^{2}(x-1)^{2}-x$, and also $U_{3}$ is a polynomial of degree 6 in $x$. When we try to determine $U_{n+1}$ inductively, we realize that the right-hand side of the recurrence formula is always divisible by $U_{n-1}$, and as a result $U_{n+1}$ becomes a polynomial of degree $n(n+1)$. Although (1.1) is of rational nature, we see that this recurrence formula is special in the sense that it produces polynomials only. Since $U_{n}$ is of degree $n(n-1)$, the degree grows rapidly and $U_{n}$ are gigantic polynomials already for small $n$.

Note that, if $f(x)$ is a polynomial in $x$ of degree $\leqslant d$, it can be written as $f(x)=F(x+1, x-1)$ by a unique homogenous polynomial $F(u, v)$ in $(u, v)$ of degree $d$; in fact, $F(u, v)$ is determined by $F(u, v)=y^{d} f(x / y)=$ $\left(\frac{u-v}{2}\right)^{d} f\left(\frac{u+v}{u-v}\right)$. If we rewrite $U_{n}$ as a homogenous polynomial of $u=x+1$ and $v=x-1$ of degree $n(n-1)$, we see that $U_{n}$ can be expressed in a concise form, and that something more interesting is happening.

$$
\begin{aligned}
U_{2} & =-\frac{1}{4}(2 a-1)(2 a+1)(x+1)^{2}+\frac{1}{4}(2 b-1)(2 b+1)(x-1)^{2} \\
& =\frac{1}{2^{2}}\left(-(c-1)(c+1) u^{2}+(d-1)(d+1) v^{2}\right),
\end{aligned}
$$

where we have set $2 a=c$ and $2 b=d$. Similarly, we have

$$
\begin{aligned}
& 2^{6} U_{3}=-(c-3)(c-1)^{2}(c+1)^{2}(c+3) u^{6} \\
& +3(c-3)(c-1)(c+1)(c+3)(d-1)(d+1) u^{4} v^{2} \\
& -3(c-1)(c+1)(d-3)(d-1)(d+1)(d+3) u^{2} v^{4} \\
& +(d-3)(d-1)^{2}(d+1)^{2}(d+3) v^{6} .
\end{aligned}
$$

We now introduce bilateral shifted factorials

$$
c_{k}=\prod_{i=0}^{k-1}(c-k+1+2 i), \quad d_{k}=\prod_{i=0}^{k-1}(d-k+1+2 i) \quad(k=0,1,2, \ldots),
$$

so that

$$
\begin{aligned}
& c_{0}=1, \quad c_{1}=c, \quad c_{2}=(c-1)(c+1), \quad c_{3}=(c-2) c(c+2), \\
& c_{4}=(c-3)(c-1)(c+1)(c+3), \quad \ldots
\end{aligned}
$$


(This definition of $c_{k}$ is different from that of [10].) Then we have

$$
\begin{aligned}
& 2^{2} U_{2}=-c_{2} u^{2}+d_{2} v^{2} \\
& 2^{6} U_{3}=-c_{4} c_{2} u^{6}+3 c_{4} d_{2} u^{4} v^{2}-3 c_{2} d_{4} u^{2} v^{4}+d_{4} d_{2} v^{6} .
\end{aligned}
$$

The coefficients $1,3,3,1$ are binomial coefficients. If we proceed to the next step,

$$
\begin{aligned}
& 2^{12} U_{4}=c_{6} c_{4} c_{2} u^{12}-6 c_{6} c_{4} d_{2} u^{10} v^{2}+ 15 c_{6} c_{2} d_{4} u^{8} v^{4} \\
&-10 c_{6} d_{4} d_{2} u^{6} v^{6}-10 c_{4} c_{2} d_{6} u^{6} v^{6}+15 c_{4} d_{6} d_{2} u^{4} v^{8} \\
&-6 c_{2} d_{6} d_{4} u^{2} v^{10}+d_{6} d_{4} d_{2} v^{12}
\end{aligned}
$$

where the binomial coefficient $\left(\begin{array}{l}6 \\ 3\end{array}\right)=20$ has split into two factorized terms with the same monomial $u^{6} v^{6}$. Let's check one more:

$$
\begin{aligned}
2^{20} U_{5}= & c_{8} c_{6} c_{4} c_{2} u^{20}-10 c_{8} c_{6} c_{4} d_{2} u^{18} v^{2}+45 c_{8} c_{6} c_{2} d_{4} u^{16} v^{4} \\
& -50 c_{8} c_{6} d_{4} d_{2} u^{14} v^{6}-70 c_{8} c_{4} c_{2} d_{6} u^{14} v^{6}+175 c_{8} c_{4} d_{6} d_{2} u^{12} v^{8} \\
& +35 c_{6} c_{4} c_{2} d_{8} u^{12} v^{8}-126 c_{8} c_{2} d_{6} d_{4} u^{10} v^{10}-126 c_{6} c_{4} d_{8} d_{2} u^{10} v^{10} \\
& +35 c_{8} d_{6} d_{4} d_{2} u^{8} v^{12}+175 c_{6} c_{2} d_{8} d_{4} u^{8} v^{12}-70 c_{6} d_{8} d_{4} d_{2} u^{6} v^{14} \\
& -50 c_{4} c_{2} d_{8} d_{6} u^{6} v^{14}+45 c_{4} d_{8} d_{6} d_{2} u^{4} v^{16} \\
& -10 c_{2} d_{8} d_{6} d_{4} u^{2} v^{18}+d_{8} d_{6} d_{4} d_{2} v^{20}
\end{aligned}
$$

Seeing these formulas, one would expect that the general formula could be

$$
2^{(n+1) n} U_{n+1}=\sum_{I \sqcup J=\{2,4, \ldots, 2 n\}}(-1)^{\frac{1}{2} \sum_{i \in I}{ }^{i}} n_{I, J} \prod_{i \in I} c_{i} u^{i} \prod_{j \in J} d_{j} v^{j}
$$

with some positive integers $n_{I, J}$, where the summation is taken over all divisions of the set $\{2,4, \ldots, 2 n\}$ into the disjoint union of two subsets.

A remarkable fact about these Umemura polynomials $U_{n+1}$ is that each coefficient $n_{I, J}$ in (1.9) represents the dimension of an irreducible polynomial representation of $\mathrm{GL}(n+1)$. Recall that the irreducible polynomial representations of the general linear group GL $(n+1)$ are parametrized by the partitions $\lambda$ with $l(\lambda) \leqslant n+1$, i.e. non-increasing sequences of nonnegative integers

$$
\lambda=\left(\lambda_{1}, \lambda_{2}, \ldots, \lambda_{n+1}\right), \quad \lambda_{1} \geqslant \lambda_{2} \geqslant \cdots \geqslant \lambda_{n+1} \geqslant 0 .
$$

For each partition $\lambda$, we denote by $L_{\mathrm{GL}(n+1)}(\lambda)$ the irreducible representation of highest weight $\lambda$. Then the dimension of $L_{\mathrm{GL}(n+1)}(\lambda)$ is given by the hooklength formula

$$
\operatorname{dim}_{\mathbb{C}} L_{\mathrm{GL}(n+1)}(\lambda)=\prod_{s \in \lambda} \frac{n+1+c_{\lambda}(s)}{h_{\lambda}(s)}
$$


where for each box $s=(i, j)$ in the Young diagram $\lambda, c_{\lambda}(s)=j-i$ and $h_{\lambda}(s)=\lambda_{i}-i+\lambda_{j}^{\prime}-j+1$ denote the content and the hook-length of $s$ respectively (for the combinatorial terminology for partitions, see Macdonald's book [7]). Returning to the formula (1.9), let $(I, J)$ be a pair of subsets of $\{2,4, \ldots, 2 n\}$ such that $I \sqcup J=\{2,4, \ldots, 2 n\}$. We express the subsets $I$ and $J$ in the form $I=\left\{2 i_{1}, 2 i_{2}, \ldots, 2 i_{r}\right\}, J=\left\{2 j_{1}, 2 j_{2}, \ldots, 2 j_{s}\right\}$ by two strict partitions $i_{1}>\cdots>i_{r}$ and $j_{1}>\cdots>j_{s}$ such that $\left\{i_{1}, \ldots, i_{r}\right\} \sqcup\left\{j_{1}, \ldots, j_{s}\right\}=$ $\{1, \ldots, n\}$. Then, to the strict partition $i_{1}>i_{2}>\cdots>i_{r}$ we attach a partition

$$
\lambda_{I}=\left(i_{1}-1, \ldots, i_{r}-1 \mid i_{1}, i_{2}, \ldots, i_{r}\right)
$$

in terms of the Frobenius symbol. The Frobenius symbol $\lambda=\left(a_{1}, \ldots, a_{r}\right.$ $\left.b_{1}, \ldots, b_{r}\right)$ with $a_{1}>\cdots>a_{r} \geqslant 0, b_{1}>\cdots>b_{r} \geqslant 0$, represents the partition $\lambda=\left(\lambda_{1}, \lambda_{2}, \ldots\right)$ such that $\lambda_{i}-i=a_{i}$ for $i=1, \ldots, r$, and $\lambda_{j}^{\prime}-j=b_{j}$ for $j=1, \ldots, r$. The observation made by [10] was that

$$
n_{I, J}=\operatorname{dim}_{\mathbb{C}} L_{\mathrm{GL}(n+1)}\left(\lambda_{I}\right)=\operatorname{dim}_{\mathbb{C}} L_{\mathrm{GL}(n+1)}\left(\lambda_{J}\right) .
$$

As an example, let's look at the coefficient of $c_{8} c_{4} d_{6} d_{2} u^{12} v^{8}$ in (1.8) for $2^{20} U_{5}$. In this case, $n=4, I=\{8,4\}, r=2$. Since $\left(i_{1}, i_{2}\right)=(4,2)$, the corresponding partition is $\lambda_{I}=(3,1 \mid 4,2)=(4,3,2,2,1)$.

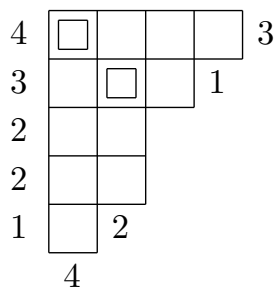

$$
\lambda_{I}=(3,1 \mid 4,2)=(4,3,2,2,1)
$$

Then the dimension of $L_{\mathrm{GL}(5)}\left(\lambda_{I}\right)$ is computed by the hook-length formula (1.11) as

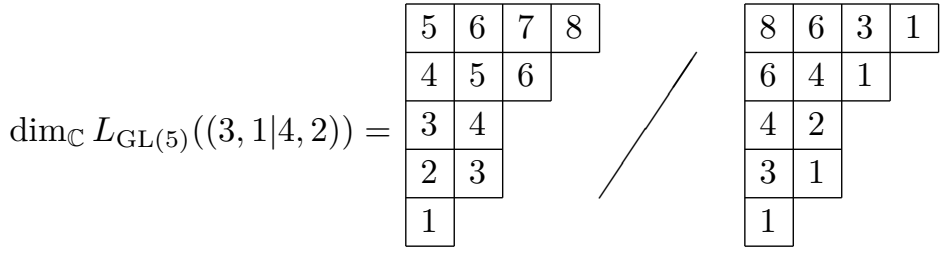

$$
\begin{aligned}
& =5 \cdot 5 \cdot 7=175 \text {. }
\end{aligned}
$$

The conjecture (1.9), (1.13) of [10] proposed by Soichi Okada, Kazuo Okamoto, Hiroshi Umemura and the author has been proved by Taneda [15]. On the basis of this work, Kirillov-Taneda [4, 5] investigated a generalization of Umemura polynomials from the combinatorial point of view. However, it seems that they did not specify how their generalized Umemura polynomials 
should be related to the sixth Painlevé equation. In the present paper, after reviewing the affine Weyl group symmetry of $P_{\mathrm{VI}}$, we formulate a precise conjecture concerning combinatorial formula for Umemura polynomials associated with a larger class of algebraic solutions of $P_{\mathrm{VI}}$ with two discrete parameters in the spirit of Kirillov-Taneda.

\section{Symmetry of the sixth Painlevé equation}

In this section, we give a summary of basic facts concerning the discrete symmetry of the sixth Painlevé equation.

The sixth Painlevé equation

$$
\begin{aligned}
\left(P_{\mathrm{VI}}\right) \frac{\mathrm{d}^{2} y}{\mathrm{~d} t^{2}}= & \frac{1}{2}\left(\frac{1}{y}+\frac{1}{y-1}+\frac{1}{y-t}\right)\left(\frac{\mathrm{d} y}{\mathrm{~d} t}\right)^{2}-\left(\frac{1}{t}+\frac{1}{t-1}+\frac{1}{y-t}\right) \frac{\mathrm{d} y}{\mathrm{~d} t} \\
& +\frac{y(y-1)(y-t)}{t^{2}\left(t^{2}-1\right)}\left(\alpha+\beta \frac{t}{y^{2}}+\gamma \frac{t-1}{(y-1)^{2}}+\delta \frac{t(t-1)}{(y-t)^{2}}\right)
\end{aligned}
$$

for $y=y(t)$ is expressed as a Hamiltonian system

$$
\left(H_{\mathrm{VI}}\right) \quad \frac{\mathrm{d} q}{\mathrm{~d} t}=\frac{\partial H}{\partial p}, \quad \frac{\mathrm{d} p}{\mathrm{~d} t}=-\frac{\partial H}{\partial q}
$$

with Hamiltonian $H=H(q, p ; t)$ defined by

$$
\begin{aligned}
t(t-1) H= & p^{2} q(q-1)(q-t) \\
& -p\left\{\left(\alpha_{0}-1\right) q(q-1)+\alpha_{3} q(q-t)+\alpha_{4}(q-1)(q-t)\right\} \\
& +\alpha_{2}\left(\alpha_{1}+\alpha_{2}\right)(q-t),
\end{aligned}
$$

or explicitly,

$$
\begin{aligned}
t(t-1) \frac{\mathrm{d} q}{\mathrm{~d} t}= & 2 p q(q-1)(q-t)-\left(\alpha_{0}-1\right) q(q-1) \\
& -\alpha_{3} q(q-t)-\alpha_{4}(q-1)(q-t) \\
t(t-1) \frac{\mathrm{d} p}{\mathrm{~d} t}= & -p^{2}((q-1)(q-t)+q(q-t)+q(q-1)) \\
& +p\left\{\left(\alpha_{0}-1\right)(2 q-1)+\alpha_{3}(2 q-t)+\alpha_{4}(2 q-1-t)\right\} \\
& -\alpha_{2}\left(\alpha_{1}+\alpha_{2}\right) .
\end{aligned}
$$

Here we use the complex parameters $\alpha_{0}, \alpha_{1}, \alpha_{2}, \alpha_{3}, \alpha_{4}$ subject to the linear relation $\alpha_{0}+\alpha_{1}+2 \alpha_{2}+\alpha_{3}+\alpha_{4}=1$. One can directly verify that the Hamiltonian system $\left(H_{\mathrm{VI}}\right)$ is equivalent to the sixth Painlevé equation $\left(P_{\mathrm{VI}}\right)$ for $y(t)=q(t)$ with parameters

$$
\alpha=\frac{1}{2} \alpha_{1}^{2}, \quad \beta=-\frac{1}{2} \alpha_{4}^{2}, \quad \gamma=\frac{1}{2} \alpha_{3}^{2}, \quad \delta=\frac{1}{2}\left(1-\alpha_{0}^{2}\right) .
$$


For two rational functions $\varphi, \psi$ in $(q, p, t)$, we define their Poisson bracket by

$$
\{\varphi, \psi\}=\frac{\partial \varphi}{\partial p} \frac{\partial \psi}{\partial q}-\frac{\partial \varphi}{\partial q} \frac{\partial \psi}{\partial p},
$$

so that $\{p, q\}=1,\{q, t\}=0,\{p, t\}=0$. Then, (2.2) implies that, for any $\varphi=\varphi(q, p, t)$, the differential equation to be satisfied by $\varphi$ is expressed as

$$
\frac{\mathrm{d} \varphi}{\mathrm{d} t}=\{H, \varphi\}+\frac{\partial \varphi}{\partial t} .
$$

We introduce parameters $\varepsilon_{1}, \varepsilon_{2}, \varepsilon_{3}, \varepsilon_{4}$ such that

$$
\begin{gathered}
\alpha_{0}=1-\varepsilon_{1}-\varepsilon_{2}, \quad \alpha_{1}=\varepsilon_{1}-\varepsilon_{2}, \quad \alpha_{2}=\varepsilon_{2}-\varepsilon_{3}, \\
\alpha_{3}=\varepsilon_{3}-\varepsilon_{4}, \quad \alpha_{4}=\varepsilon_{3}+\varepsilon_{4}
\end{gathered}
$$

and regard $\varepsilon=\left(\varepsilon_{1}, \varepsilon_{2}, \varepsilon_{3}, \varepsilon_{4}\right)$ as the coordinates of the four dimensional affine space $V=\mathbb{C}^{4}$. We identify this parameter space $V$ with the Cartan subalgebra of the simple Lie algebra $\mathfrak{s o}(8)$ of type $D_{4}$, and regard the parameters $\alpha_{j}(j=0,1,2,3,4)$ as the simple affine roots of type $D_{4}^{(1)}$ identifying the null root $\delta=\alpha_{0}+\alpha_{1}+2 \alpha_{2}+\alpha_{3}+\alpha_{4}$ with the constant function 1 on $V$.

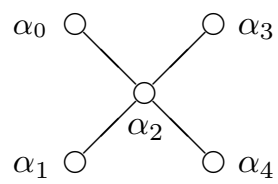

It has been known since the pioneering work of Kazuo Okamoto [14] that the Hamiltonian system $\left(H_{\mathrm{VI}}\right)$ admits a group of Bäcklund transformations which is isomorphic to the extended affine Weyl group $\widetilde{W}\left(D_{4}^{(1)}\right)$ of type $D_{4}$. This group $\widetilde{W}\left(D_{4}^{(1)}\right)=\left\langle s_{0}, s_{1}, s_{2}, s_{3}, s_{4} ; r_{1}, r_{3}, r_{4}\right\rangle$ is generated by simple reflections $s_{i}(i=0,1, \ldots, 4)$ attached to the simple affine roots $\alpha_{i}$ and the Dynkin diagram automorphisms $r_{1}, r_{3}$ and $r_{4}=r_{1} r_{3}$ corresponding to the permutations $\sigma_{1}=(01)(34), \sigma_{3}=(03)(14), \sigma_{4}=(04)(13)$ of $\{0,1,3,4\}$ : these generators are subject to the fundamental relations

$$
\begin{aligned}
& s_{i}^{2}=1 \quad(i=0,1, \ldots, 4) ; \quad s_{i} s_{j}=s_{j} s_{i} \quad(i, j=0,1,3,4) ; \quad s_{i} s_{2} s_{i}=s_{2} s_{i} s_{2} \\
& (i=0,1,3,4)
\end{aligned}
$$

and

$$
\begin{aligned}
r_{i}^{2}=1 \quad(i=1,3) ; \quad r_{1} r_{3}=r_{3} r_{1} ; \quad r_{k} s_{i}= & s_{\sigma_{k}(i)} r_{k} \\
& (k=1,3: i=0,1,3,4) .
\end{aligned}
$$


Table 2.1. Fundamental Bäcklund transformations of $\left(H_{\mathrm{VI}}\right)$

\begin{tabular}{|c||c|c|c|c|c||c|c|}
\hline & $\alpha_{0}$ & $\alpha_{1}$ & $\alpha_{2}$ & $\alpha_{3}$ & $\alpha_{4}$ & $q$ & $p$ \\
\hline \hline$s_{0}$ & $-\alpha_{0}$ & $\alpha_{1}$ & $\alpha_{2}+\alpha_{0}$ & $\alpha_{3}$ & $\alpha_{4}$ & $q$ & $p-\frac{\alpha_{0}}{q-t}$ \\
\hline$s_{1}$ & $\alpha_{0}$ & $-\alpha_{1}$ & $\alpha_{2}+\alpha_{1}$ & $\alpha_{3}$ & $\alpha_{4}$ & $q$ & $p$ \\
\hline$s_{2}$ & $\alpha_{0}+\alpha_{2}$ & $\alpha_{1}+\alpha_{2}$ & $-\alpha_{2}$ & $\alpha_{3}+\alpha_{2}$ & $\alpha_{4}+\alpha_{2}$ & $q+\frac{\alpha_{2}}{p}$ & $p$ \\
\hline$s_{3}$ & $\alpha_{0}$ & $\alpha_{1}$ & $\alpha_{2}+\alpha_{3}$ & $-\alpha_{3}$ & $\alpha_{4}$ & $q$ & $p-\frac{\alpha_{3}}{q-1}$ \\
\hline$s_{4}$ & $\alpha_{0}$ & $-\alpha_{1}$ & $\alpha_{2}+\alpha_{4}$ & $\alpha_{3}$ & $-\alpha_{4}$ & $q$ & $p-\frac{\alpha_{4}}{q}$ \\
\hline \hline$r_{1}$ & $\alpha_{1}$ & $\alpha_{0}$ & $\alpha_{2}$ & $\alpha_{4}$ & $\alpha_{3}$ & $\frac{t(q-1)}{q-t}$ & $-\frac{(q-t)\left((q-t) p+\alpha_{2}\right)}{t(t-1)}$ \\
\hline$r_{3}$ & $\alpha_{3}$ & $\alpha_{4}$ & $\alpha_{2}$ & $\alpha_{0}$ & $\alpha_{1}$ & $\frac{t}{q}$ & $-\frac{q\left(q p+\alpha_{2}\right)}{t}$ \\
\hline$r_{4}$ & $\alpha_{4}$ & $\alpha_{3}$ & $\alpha_{2}$ & $\alpha_{1}$ & $\alpha_{0}$ & $\frac{q-t}{q-1}$ & $\frac{(q-1)\left((q-1) p+\alpha_{2}\right)}{t-1}$ \\
\hline
\end{tabular}

Let $\mathcal{K}=\mathbb{C}(\alpha ; q, p, t)$ the field of rational functions in $\left(\alpha_{1}, \alpha_{2}, \alpha_{3}, \alpha_{4} ; q, p, t\right)$ and define the derivation $\partial_{t}=\mathrm{d} / \mathrm{d} t: \mathcal{K} \rightarrow \mathcal{K}$ by

$$
\begin{aligned}
\partial_{t}\left(\alpha_{i}\right)=0 \quad(i=0,1, \ldots, 4) ; \\
\partial_{t}(q)=\frac{\partial H}{\partial p}, \quad \partial_{t}(p)=-\frac{\partial H}{\partial q}, \quad \partial_{t}(t)=1 .
\end{aligned}
$$

By a Bäcklund transformation of $\left(H_{\mathrm{VI}}\right)$, we mean an automorphism $w$ of the differential field $\left(\mathcal{K}, \partial_{t}\right)$, i.e. a $\mathbb{C}$-automorphism $w: \mathcal{K} \rightarrow \mathcal{K}$ of the field that commutes with $\partial_{t}: w\left(\partial_{t}(\varphi)\right)=\partial_{t}(w(\varphi))$ for all $\varphi \in \mathcal{K}$. If we regard an automorphism of $\mathcal{K}$ as representing a birational mapping of the seven dimensional affine space with coordinates $\left(\varepsilon_{1}, \varepsilon_{2}, \varepsilon_{3}, \varepsilon_{4} ; q, p, t\right)$, then a Bäcklund transformation is a birational transformation which leaves the differential equation $\left(H_{\mathrm{VI}}\right)$ invariant.

The Hamiltonian system $\left(H_{\mathrm{VI}}\right)$ has eight fundamental Bäcklund transformations $s_{0}, s_{1}, s_{2}, s_{3}, s_{4}$ and $r_{1}, r_{3}, r_{4}$ as specified in Table 2.1 in terms of the action on the generators of $\mathcal{K}$. Note also that these transformations preserve the Poisson bracket, namely $w(\{\varphi, \psi\})=\{w(\varphi), w(\psi)\}$ for all $\varphi, \psi \in \mathcal{K}$.

We give below some comments on how to find these Bäcklund transformations. Leaving this question aside, one can directly verify that these birational transformations leave $\left(H_{\mathrm{VI}}\right)$ invariant, and also that they satisfy the fundamental relations $(2.10),(2.11)$ of the generators of the extended affine Weyl group $\widetilde{W}\left(D_{4}^{(1)}\right)$. We denote by $\widetilde{W}=\left\langle s_{0}, s_{1}, s_{2}, s_{3}, s_{4}, r_{1}, r_{3}, r_{4}\right\rangle \subset \operatorname{Aut}\left(\mathcal{K}, \partial_{t}\right)$ the group of Bäcklund transformations generated by $s_{i}$ and $r_{i}$. Note that the action of $\widetilde{W}$ stabilizes the subfield $\mathbb{C}(\alpha) \subset \mathcal{K}$, and is identical to the action of 
$\widetilde{W}\left(D_{4}^{(1)}\right)$ on the field of rational functions of $V$ induced from its standard action on the affine space $V$. In what follows, we denote by $\langle\cdot, \cdot\rangle: V^{*} \times V^{*} \rightarrow \mathbb{C}$ the scalar product (symmetric $\mathbb{C}$-bilinear form) defined by $\left\langle\varepsilon_{i}, \varepsilon_{j}\right\rangle=\delta_{i, j}$ $(i, j=1, \ldots, 4)$. Also, we use the notation

$$
Q=\mathbb{Z} \alpha_{1} \oplus \mathbb{Z} \alpha_{2} \oplus \mathbb{Z} \alpha_{3} \oplus \mathbb{Z} \alpha_{4} \subset P=\mathbb{Z} \omega_{1} \oplus \mathbb{Z} \omega_{2} \oplus \mathbb{Z} \omega_{3} \oplus \mathbb{Z} \omega_{4} \subset V^{*}
$$

the root lattice and the weight lattice of type $D_{4}$; the fundamental weights are specified as

$\omega_{1}=\varepsilon_{1}, \quad \omega_{2}=\varepsilon_{1}+\varepsilon_{2}, \quad \omega_{3}=\frac{1}{2}\left(\varepsilon_{1}+\varepsilon_{2}+\varepsilon_{3}-\varepsilon_{4}\right), \quad \omega_{4}=\frac{1}{2}\left(\varepsilon_{1}+\varepsilon_{2}+\varepsilon_{3}+\varepsilon_{4}\right)$.

For each $\mu \in P$, we define the translation $T_{\mu}: \mathbb{C}(\alpha) \rightarrow \mathbb{C}(\alpha)$ by $\mu$ as the $\mathbb{C}$ automorphism such that $T_{\mu}\left(\varepsilon_{i}\right)=\alpha-\left\langle\mu, \varepsilon_{i}\right\rangle(i=1,2,3,4)$. Then we obtain the two groups of translations

$$
T(Q)=\left\{T_{\alpha} \mid \alpha \in Q\right\} \subset T(P)=\left\{T_{\mu} \mid \mu \in P\right\} \subset \operatorname{Aut}(\mathbb{C}(\alpha))
$$

by the root lattice and by the weight lattice; both $T(Q)$ and $T(P)$ are free abelian groups of rank 4 . It is known that $T(P)$ is contained in the extended affine Weyl group $\widetilde{W}=\left\langle s_{0}, s_{1}, s_{2}, s_{3}, s_{4} ; r_{1}, r_{3}, r_{4}\right\rangle$, and $\widetilde{W}$ is expressed as the semidirect product $\widetilde{W}=T(P) \rtimes W_{0}$ of $T(P)$ and the finite Weyl group $W_{0}=W\left(D_{4}\right)=\left\langle s_{1}, s_{2}, s_{3}, s_{4}\right\rangle$ acting on $T(P)$. We remark that the subgroup $W=\left\langle s_{0}, s_{1}, s_{2}, s_{3}, s_{4}\right\rangle \subset \widetilde{W}$ is the standard affine Weyl group $W\left(D_{4}^{(1)}\right)$, and is expressed as the semidirect product $W=T(Q) \rtimes W_{0}$. In terms of the generators of $\widetilde{W}$, the translations by the simple roots and the fundamental weights are expressed as follows.

$$
\begin{array}{ll}
T_{\alpha_{1}}=s_{2} s_{0} s_{3} s_{4} s_{2} s_{0} s_{3} s_{4} s_{2} s_{1} & T_{\omega_{1}}=s_{0} s_{2} s_{3} s_{4} s_{2} s_{0} r_{1} \\
T_{\alpha_{2}}=s_{0} s_{1} s_{3} s_{4} s_{2} s_{0} s_{1} s_{3} s_{4} s_{2} & T_{\omega_{2}}=s_{0} s_{2} s_{1} s_{3} s_{4} s_{2} s_{1} s_{3} s_{4} s_{2} \\
T_{\alpha_{3}}=s_{2} s_{0} s_{1} s_{4} s_{2} s_{0} s_{1} s_{4} s_{2} s_{3} & T_{\omega_{3}}=s_{0} s_{2} s_{1} s_{4} s_{2} s_{0} r_{3} \\
T_{\alpha_{4}}=s_{2} s_{0} s_{1} s_{3} s_{2} s_{0} s_{1} s_{3} s_{2} s_{4} & T_{\omega_{4}}=s_{0} s_{2} s_{1} s_{3} s_{2} s_{0} r_{4} .
\end{array}
$$

We already know that the action of the extended Weyl group $\widetilde{W}=$ $\left\langle s_{0}, s_{1}, s_{2}, s_{3}, s_{4} ; r_{1}, r_{3}, r_{4}\right\rangle$ on $\mathbb{C}(\alpha)$ can be lifted to $\mathcal{K}=\mathbb{C}(\alpha ; q, p, t)$ preserving the fundamental relations of the generators. From the representation $\widetilde{W}=T(P) \rtimes W_{0}$, we obtain a commutative family $T_{\mu}(\mu \in P)$ of Bäcklund transformations parametrized by the weight lattice. These translations $T_{\mu}: \mathcal{K} \rightarrow \mathcal{K}$ are also called the Schlesinger transformations.

We remark that, besides this transformation group $\widetilde{W} \subset \operatorname{Aut}\left(\mathcal{K}, \partial_{t}\right)$, the Hamiltonian system $\left(H_{\mathrm{VI}}\right)$ has birational canonical transformations (that leave the 2-form $\Omega=\mathrm{d} p \wedge \mathrm{d} q-\mathrm{d} H \wedge \mathrm{d} t$ invariant) corresponding to the permutation group $\mathfrak{S}_{\{0,1,3,4\}}$ of the indexing set $\{0,1,3,4\}$. We describe in Table 2.2 the birational canonical transformations $\sigma_{i j}$ corresponding to the 
Table 2.2. Birational canonical transformations that realize $\mathfrak{S}_{\{0,1,3,4\}}$

\begin{tabular}{|c||c|c|c|c|c||c|c|c|c|}
\hline & $\alpha_{0}$ & $\alpha_{1}$ & $\alpha_{2}$ & $\alpha_{3}$ & $\alpha_{4}$ & $t$ & $\partial_{t}$ & $q$ & $p$ \\
\hline \hline$\sigma_{01}$ & $\alpha_{1}$ & $\alpha_{0}$ & $\alpha_{2}$ & $\alpha_{3}$ & $\alpha_{4}$ & $1-t$ & $-\partial_{t}$ & $\frac{(1-t) q}{q-t}$ & $\frac{(q-t)\left((q-t) p+\alpha_{2}\right)}{t((t-1)}$ \\
\hline$\sigma_{03}$ & $\alpha_{3}$ & $\alpha_{1}$ & $\alpha_{2}$ & $\alpha_{0}$ & $\alpha_{4}$ & $\frac{1}{t}$ & $-t^{2} \partial_{t}$ & $\frac{q}{t}$ & $t p$ \\
\hline$\sigma_{04}$ & $\alpha_{4}$ & $\alpha_{1}$ & $\alpha_{2}$ & $\alpha_{3}$ & $\alpha_{0}$ & $\frac{t}{t-1}$ & $-(t-1)^{2} \partial_{t}$ & $\frac{q-t}{1-t}$ & $(1-t) p$ \\
\hline$\sigma_{13}$ & $\alpha_{0}$ & $\alpha_{3}$ & $\alpha_{2}$ & $\alpha_{1}$ & $\alpha_{4}$ & $\frac{t}{t-1}$ & $-(t-1)^{2} \partial_{t}$ & $\frac{q}{q-1}$ & $-(q-1)\left((q-1) p+\alpha_{2}\right)$ \\
\hline$\sigma_{14}$ & $\alpha_{0}$ & $\alpha_{4}$ & $\alpha_{2}$ & $\alpha_{3}$ & $\alpha_{1}$ & $\frac{1}{t}$ & $-t^{2} \partial_{t}$ & $\frac{1}{q}$ & $-q\left(q p+\alpha_{2}\right)$ \\
\hline$\sigma_{34}$ & $\alpha_{0}$ & $\alpha_{1}$ & $\alpha_{2}$ & $\alpha_{4}$ & $\alpha_{3}$ & $1-t$ & $-\partial_{t}$ & $1-q$ & $-p$ \\
\hline
\end{tabular}

transpositions $(i j) \in \mathfrak{S}_{\{0,1,3,4\}}$. Notice that the Bäcklund transformations $r_{1}, r_{3}, r_{4}$ are obtained from these birational canonical transformations as compositions $r_{1}=\sigma_{01} \sigma_{34}, r_{3}=\sigma_{03} \sigma_{14}, r_{4}=\sigma_{04} \sigma_{13}$. If we include these birational canonical transformations that represent $\mathfrak{S}_{\{0,1,3,4\}}$ as well, the symmetry group of $\left(H_{\mathrm{VI}}\right)$ extends to an affine Weyl group of type $F_{4}$.

Recall that the Hamiltonian system $\left(H_{\mathrm{VI}}\right)$ arises from the monodromy preserving deformation

$$
\begin{gathered}
\left(\partial_{z}^{2}+a_{1}(z ; t) \partial_{z}+a_{2}(z ; t)\right) u(z ; t)=0, \\
\partial_{t} u(z ; t)=\left(b_{1}(z ; t) \partial_{z}+b_{2}(z ; t)\right) u(z ; t)
\end{gathered}
$$

of a second order Fuchsian differential equation for $u=u(z ; t)$ on $\mathbb{P}^{1}$ with four regular singular points and an apparent singularity, where $\partial_{z}=\partial / \partial z$ and $\partial_{t}=\partial / \partial t$. The first equation is assumed to be Fuchsian with Riemann scheme

$$
\left\{\begin{array}{ccccc}
z=0 & z=1 & z=t & z=q & z=\infty \\
0 & 0 & 0 & 0 & \alpha_{2} \\
\alpha_{4} & \alpha_{3} & \alpha_{0} & 2 & \alpha_{1}+\alpha_{2}
\end{array}\right\},
$$

and the singularity $z=q$ with characteristic exponents 0,2 , which may depend on $t$, is assumed to be non-logarithmic. Under these assumptions, the coefficients $a_{1}(z ; t)$ and $a_{2}(z ; t)$ are determined uniquely by $p=$ 
$\operatorname{Res}_{z=q}\left(a_{2}(z ; t) \mathrm{d} z\right)$ as

$$
\begin{aligned}
& a_{1}(z ; t)=\frac{1-\alpha_{4}}{z}+\frac{1-\alpha_{3}}{z-1}+\frac{1-\alpha_{0}}{z-t}-\frac{1}{z-q}, \\
& a_{2}(z ; t)=\frac{1}{z(z-1)}\left(-\frac{t(t-1) H}{z-t}+\frac{q(q-1) p}{z-q}+\alpha_{2}\left(\alpha_{1}+\alpha_{2}\right)\right),
\end{aligned}
$$

In particular the Hamiltonian is obtained as $H=-\operatorname{Res}_{z=t}\left(a_{2}(z ; t) \mathrm{d} z\right)$. The compatibility condition of (2.17) is then equivalent to the Hamiltonian system $\left(H_{\mathrm{VI}}\right)$. In this context of monodromy preserving deformation, the Bäcklund transformations $s_{0}, s_{1}, s_{3}, s_{4}$ except $s_{2}$ arise from gauge transformations $u(z ; t) \rightarrow g(z ; t) u(z ; t)$ by power functions in $z$ branching only at $z=0,1, t, \infty$. Also, the birational canonical transformations of Table 2.2 are obtained from the coordinate changes in $z$ by fractional linear transformations that permutes the singular points $\{0,1, t, \infty\}$.

Where does the fundamental Bäcklund transformation $s_{2}$ come from? We give a comment on how Okamoto obtained nontrivial Bäcklund transformations, recalling some of the key ideas of [14]. Incidentally, $s_{2}$ is sometimes called the Okamoto transformation. Okamoto renormalizes the Hamiltonian by setting

$$
\begin{aligned}
h & =t(t-1) H(q, p ; t)+c_{1} t+c_{0}, \\
c_{1} & =\varepsilon_{1} \varepsilon_{2}-\varepsilon_{1} \varepsilon_{3}-\varepsilon_{2} \varepsilon_{3}, \\
c_{0} & =-\frac{1}{2}\left(\varepsilon_{1} \varepsilon_{2}-\varepsilon_{1} \varepsilon_{3}-\varepsilon_{1} \varepsilon_{4}-\varepsilon_{2} \varepsilon_{3}-\varepsilon_{2} \varepsilon_{4}+\varepsilon_{3} \varepsilon_{4}\right),
\end{aligned}
$$

and shows that the Hamiltonian system $\left(H_{\mathrm{VI}}\right)$ is equivalent to a single differential equation

$$
\begin{aligned}
\frac{\mathrm{d} h}{\mathrm{~d} t}\left(t(t-1) \frac{\mathrm{d}^{2} h}{\mathrm{~d} t}\right)^{2} & +\left(2 h \frac{\mathrm{d} h}{\mathrm{~d} t}-(2 t-1)\left(\frac{\mathrm{d} h}{\mathrm{~d} t}\right)^{2}+\varepsilon_{1} \varepsilon_{2} \varepsilon_{3} \varepsilon_{4}\right)^{2} \\
= & \left(\frac{\mathrm{d} h}{\mathrm{~d} t}+\varepsilon_{1}^{2}\right)\left(\frac{\mathrm{d} h}{\mathrm{~d} t}+\varepsilon_{2}^{2}\right)\left(\frac{\mathrm{d} h}{\mathrm{~d} t}+\varepsilon_{3}^{2}\right)\left(\frac{\mathrm{d} h}{\mathrm{~d} t}+\varepsilon_{4}^{2}\right)
\end{aligned}
$$

for the Hamiltonian function $h=h(t)$ under the assumption that $\mathrm{d}^{2} h / \mathrm{d} t^{2} \neq$ 0 . Also, if $h=h(t)$ is a generic solution of (2.21), the variables $q, p$ can be recovered from $h, h^{\prime}=\mathrm{d} h / \mathrm{d} t$ and $h^{\prime \prime}=\mathrm{d}^{2} h / \mathrm{d} t^{2}$ by the relations

$$
\begin{aligned}
2\left(h^{\prime}+\varepsilon_{1}^{2}\right)\left(h^{\prime}+\varepsilon_{2}^{2}\right) q= & -\left(\varepsilon_{1}+\varepsilon_{2}\right) t(t-1) h^{\prime \prime} \\
& +\left((2 t-1) h^{\prime}-2 h-\varepsilon_{1} \varepsilon_{2}\right)\left(h^{\prime}-\varepsilon_{1} \varepsilon_{2}\right) \\
& +\left(h^{\prime}+\varepsilon_{1}^{2}+\varepsilon_{1} \varepsilon_{2}+\varepsilon_{2}^{2}\right)\left(h^{\prime}+\varepsilon_{3} \varepsilon_{4}\right), \\
\left(\varepsilon_{1}+\varepsilon_{2}\right) q(q-1) p= & h+(q-t) h^{\prime}+c_{1} q+c_{0} .
\end{aligned}
$$

Note that the differential equation (2.21) is manifestly invariant under the action of the Weyl group $W\left(D_{4}\right)$ of type $D_{4}$ on the parameters $\varepsilon_{1}, \varepsilon_{2}, \varepsilon_{3}, \varepsilon_{4}$ (the 
permutations and the even number of sign changes of $\varepsilon_{i}$ ). (In fact Okamoto uses the coordinates $b=\left(b_{1}, b_{2}, b_{3}, b_{4}\right)$ of $V$ such that $b_{1}=\varepsilon_{3}, b_{2}=\varepsilon_{4}$, $b_{3}=-\varepsilon_{2}, b_{4}=-\varepsilon_{1}$.) Analyzing the differential equation (2.21), Okamoto then constructs birational canonical transformations lifting the simple reflections $s_{i}$ on the parameter space, as well as those lifting parallel translations on the parameters. In fact, the birational canonical transformation corresponding to $s_{2}$ (transposition of $\varepsilon_{2}, \varepsilon_{3}$ ) is determined as

$$
\begin{aligned}
& s_{2}: \quad\left(\varepsilon_{1}, \varepsilon_{2}, \varepsilon_{3}, \varepsilon_{4} ; q, p, t, H\right) \longrightarrow \\
& \left(\varepsilon_{1}, \varepsilon_{3}, \varepsilon_{2}, \varepsilon_{4} ; q+\frac{\varepsilon_{2}-\varepsilon_{3}}{p}, p, t, H+\frac{\left(\varepsilon_{2}-\varepsilon_{3}\right)\left(\varepsilon_{1}-\varepsilon_{4}\right)}{t}+\frac{\left(\varepsilon_{2}-\varepsilon_{3}\right)\left(\varepsilon_{1}+\varepsilon_{4}\right)}{t-1}\right) .
\end{aligned}
$$

As to parallel translations, Okamoto deals with the shift with respect to $\varepsilon_{2}$ as an example; the corresponding canonical transformation is given by

$$
\begin{aligned}
T_{\varepsilon_{2}}: & \left(\varepsilon_{1}, \varepsilon_{2}, \varepsilon_{3}, \varepsilon_{4} ; q, p, t, H\right) \longrightarrow \\
& \left(\varepsilon_{1}, \varepsilon_{2}-1, \varepsilon_{3}, \varepsilon_{4} ; \widetilde{q}, \widetilde{p}, t, H+\frac{\left(\varepsilon_{2}-\varepsilon_{3}\right)\left(\varepsilon_{1}-\varepsilon_{4}\right)}{t}+\frac{\left(\varepsilon_{2}-\varepsilon_{3}\right)\left(\varepsilon_{1}+\varepsilon_{4}\right)}{t-1}\right),
\end{aligned}
$$

where $\widetilde{q}, \widetilde{p}$ are rational functions in $(q, p)$ which do not specify here. Also, Okamoto constructs birational canonical transformations corresponding to the permutations of the simple affine roots $\alpha_{0}, \alpha_{1}, \alpha_{3}, \alpha_{4}$. In this way, Okamoto clarified that the transformation group of $\left(H_{\mathrm{VI}}\right)$ of birational canonical transformations that leaves $\partial_{t}$ invariant form an extension of the affine Weyl group of type $D_{4}$, and that, together with the birational canonical transformations lifting the Dynkin diagram automorphisms, this transformation group extends to an affine Weyl group of type $F_{4}$.

In order to describe the action of translations in a systematic way, Okamoto introduces the notion of $\tau$ function for the Hamiltonian system $\left(H_{\mathrm{VI}}\right)$ as a dependent variable such that

$$
H=\frac{\mathrm{d}}{\mathrm{d} t} \log \tau=\frac{1}{\tau} \frac{\mathrm{d} \tau}{\mathrm{d} t} .
$$

Note that this $\tau$ function is determined up to multiplication by a constant which may depend on the parameters. For each $m \in \mathbb{Z}$, we denote the $m$-th iteration of the birational canonical transformation (2.24) by

$$
T_{\varepsilon_{2}}^{m}: \quad\left(\varepsilon_{1}, \varepsilon_{2}, \varepsilon_{3}, \varepsilon_{4} ; q, p, t, H\right) \rightarrow\left(\varepsilon_{1}, \varepsilon_{2}-m, \varepsilon_{3}, \varepsilon_{4} ; q_{m}, p_{m}, t, H_{m}\right)
$$

and by $\tau_{m}$ the corresponding $\tau$ function. Investigating the differential equation (2.21) for the Hamiltonian function, Okamoto proves that this sequence 
of $\tau$ functions $\left(\tau_{m}\right)_{m \in \mathbb{Z}}$ satisfies the Toda equation

$$
\frac{\mathrm{d}}{\mathrm{d} t} t(t-1) \frac{\mathrm{d}}{\mathrm{d} t} \log \tau_{m}+\left(\varepsilon_{1}+\varepsilon_{2}-m\right)\left(\varepsilon_{2}-\varepsilon_{3}-m\right)=c_{m} \frac{\tau_{m-1} \tau_{m+1}}{\tau_{m}^{2}} \quad(m \in \mathbb{Z}),
$$

where $c_{m}$ are constants which depend on the renormalization of the $\tau$ functions. If we specify the initial $\tau$ functions $\tau_{0}, \tau_{1}$, in the generic setting all the other $\tau$ functions $\tau_{m}$ are determined by this Toda equation.

Remark 2.1. - In the context of the monodromy preserving deformation of second order Fuchsian differential equations, it is known that the Okamoto transformation $s_{2}$ can be interpreted in terms of an integral transformation of Euler type, called the middle convolution (see Filipuk [1], Novikov [13]; for related works by Boalch, Harnad, and Mazzocco, see Haraoka-Filipuk [2] and references therein). Also, in the framework of the $8 \times 8$ Lax pair of NoumiYamada [12], the Bäcklund transformations discussed above naturally arise from the action of the affine Weyl group associated with $\widehat{\mathfrak{s o}}(8)$.

\section{Umemura polynomials with two discrete parameters}

After his works on the irreducibility and the classification of transcendental classical solutions of the Painlevé equations ([16, 17, 18]), Hiroshi Umemura proposed around 1995 to investigate the special polynomials arising from the sequences of $\tau$ functions of the sixth Painlevé equation $([10,20])$. His interest was directed in particular to those associated with algebraic solutions appearing as fixed points of Bäcklund transformations. Special polynomials in this case can be regarded as counterparts for $P_{\mathrm{VI}}$ of the YablonskiVorobiev polynomials for $P_{\mathrm{II}}$ and the Okamoto polynomials for $P_{\mathrm{IV}}$ (see [9] for the cases of $P_{\mathrm{II}}$ and $\left.P_{\mathrm{IV}}\right)$. In this section we reproduce the construction of Umemura polynomials starting from a simpler seed solution than the one Umemura originally worked on. After that, we introduce Umemura polynomials $U_{m, n}(x ; c, d)$ with two discrete parameters following the work of Tetsu Masuda [8]. We will not touch here upon the classical solutions of hypergeometric type that appear on the walls of the affine Weyl group.

Keeping the notations of the previous section, we consider the extended affine Weyl group $\widetilde{W}=\left\langle s_{0}, s_{1}, s_{2}, s_{3}, s_{4} ; r_{1}, r_{3}, r_{4}\right\rangle$ acting on the differential field $\left(\mathcal{K}, \partial_{t}\right), \mathcal{K}=\mathbb{C}(\alpha ; q, p, t)$, as a group of Bäcklund transformations for $\left(H_{\mathrm{VI}}\right)$. As we remarked already, this transformation group can also be expressed as the semidirect product $\widetilde{W}=T(P) \rtimes W_{0}$ of the abelian group of translations by the weight lattice $P$ (Schlesinger transformations) and the 
finite Weyl group $W_{0}=W\left(D_{4}\right)=\left\{s_{1}, s_{2}, s_{3}, s_{4}\right\}$. Let $w \in \widetilde{W}$ and set

$$
\begin{aligned}
& w\left(\alpha_{i}\right)=\alpha_{w, i} \quad(i=0,1, \ldots, 4), \\
& w(q)=\psi_{w}(\alpha ; q, p, t), \quad w(p)=\varphi_{w}(\alpha ; q, p, t),
\end{aligned}
$$

where $\alpha_{w, i}$ are linear functions in $\alpha_{j}$, and $\psi_{w}(\alpha ; q, p, t), \varphi_{w}(\alpha ; q, p, t)$ are rational functions in $(q, p, t)$ with coefficients in $\mathbb{C}(\alpha)$. Let $(q(t), p(t))$ be a solution of $\left(H_{\mathrm{VI}}\right)$ with parameters $\alpha=\left(\alpha_{0}, \alpha_{1}, \alpha_{2}, \alpha_{3}, \alpha_{4}\right)$ and consider the functions

$$
q_{w}(t)=\psi_{w}(\alpha ; q(t), p(t), t), \quad p_{w}(t)=\varphi_{w}(\alpha ; q(t), p(t), t),
$$

supposing $(q(t), p(t))$ is generic in the sense that these substitutions make sense. The assumption that $w$ commutes with $\partial_{t}$ then implies that $\left(q_{w}(t), p_{w}(t)\right)$ gives rise to a solution of $\left(H_{\mathrm{VI}}\right)$ with parameters $\alpha_{w}=$ $\left(\alpha_{w, 0}, \ldots, \alpha_{w, 4}\right)$. In this way, we obtain a large class of solutions $\left(q_{w}(t), p_{w}(t)\right)$ $(w \in \widetilde{W})$ starting from a generic seed solution $(q(t), p(t))$.

Suppose now that $w \in \widetilde{W}$ has a fixed point $(\alpha ; q, p), q=q(t), p=p(t)$ :

$$
\alpha_{w, i}=\alpha_{i} \quad(i=0,1, \ldots, 4), \quad \psi_{w}(\alpha ; q, p)=q, \quad \varphi_{w}(\alpha ; q, p, t)=p .
$$

Note that the assumption that $w$ commute with $\partial_{t}$ can be written as

$$
\begin{aligned}
& \frac{\partial H}{\partial p} \frac{\partial \psi_{w}}{\partial q}-\frac{\partial H}{\partial q} \frac{\partial \psi_{w}}{\partial p}+\frac{\partial \psi_{w}}{\partial t}=w\left(\frac{\partial H}{\partial p}\right) \\
& \frac{\partial H}{\partial p} \frac{\partial \varphi_{w}}{\partial q}-\frac{\partial H}{\partial q} \frac{\partial \varphi_{w}}{\partial p}+\frac{\partial \varphi_{w}}{\partial t}=-w\left(\frac{\partial H}{\partial q}\right) .
\end{aligned}
$$

On the other hand, the assumption that $(q(t), p(t))$ is a fixed point of $w$ implies

$$
\frac{\mathrm{d} q}{\mathrm{~d} t} \frac{\partial \psi_{w}}{\partial q}-\frac{\mathrm{d} p}{\mathrm{~d} t} \frac{\partial \psi_{w}}{\partial p}+\frac{\partial \psi_{w}}{\partial t}=\frac{\mathrm{d} q}{\mathrm{~d} t}, \quad \frac{\mathrm{d} q}{\mathrm{~d} t} \frac{\partial \varphi_{w}}{\partial q}-\frac{\mathrm{d} p}{\mathrm{~d} t} \frac{\partial \varphi_{w}}{\partial p}+\frac{\partial \varphi_{w}}{\partial t}=\frac{\mathrm{d} p}{\mathrm{~d} t} .
$$

Noting that $w(\partial H / \partial p)=\partial H / \partial p, w(\partial H / \partial q)=\partial H / \partial q$ at the fixed point, and using $\left\{\varphi_{w}, \psi_{w}\right\}=1$, one can show that $(q(t), p(t))$ must be a solution of $\left(H_{\mathrm{VI}}\right)$ whenever $\partial \psi_{w} / \partial q+\partial \varphi_{w} / \partial p \neq 2$.

We now take the Dynkin diagram automorphism $r_{3}$ for $w$. From the data of Table 2.1, the condition of a fixed point of $r_{3}$ is given by

$$
\alpha_{0}=\alpha_{3}, \alpha_{1}=\alpha_{4}, \quad t / q=q, \quad-q\left(q p+\alpha_{2}\right) / t=p .
$$

Solving these equations, we see that, for the parameter values

$$
\alpha=\left(\alpha_{0}, \alpha_{1}, \alpha_{2}, \alpha_{3}, \alpha_{4}\right)=\left(a, b, \frac{1}{2}-a-b, a, b\right) \quad(a, b \in \mathbb{C}),
$$


there exist simple algebraic solutions

$$
(q(t), p(t))=\left(t^{\frac{1}{2}}, \frac{1}{2}\left(a+b-\frac{1}{2}\right) t^{-\frac{1}{2}}\right) .
$$

We investigate below the class of algebraic solutions that are obtained by a two-parameter family of Bäcklund transformations from this seed solution.

For the moment, we take the fundamental weight $\omega_{3}=\frac{1}{2}\left(\varepsilon_{1}+\varepsilon_{2}+\varepsilon_{3}-\varepsilon_{4}\right)$ and use the $m$-th iteration of the translation $T_{\omega_{3}}$ :

$$
\left(T_{\omega_{3}}^{m}\left(\alpha_{0}\right), \ldots, T_{\omega_{3}}^{m}\left(\alpha_{4}\right)\right)=\left(\alpha_{0}+m, \alpha_{1}, \alpha_{2}, \alpha_{3}-m, \alpha_{4}\right) \quad(m \in \mathbb{Z}) .
$$

Applying the Bäcklund transformation $w=T_{\omega_{3}}^{m}$ to the seed solution mentioned above, we obtain algebraic solutions $\left(q_{m}(t), p_{m}(t)\right)(m \in \mathbb{Z})$ with $\alpha$ parameters $\left(a+m, b, \frac{1}{2}-a-b, a-m, b\right)$ for each $m \in \mathbb{Z}$. For example, we first compute

$$
\begin{aligned}
& T_{\omega_{3}}(q)=\frac{t\left(p(q-t)-\alpha_{0}\right)\left(p q(q-t)-\left(\alpha_{0}+\alpha_{4}\right) q+t \alpha_{4}\right)}{\left(p q(q-t)+\alpha_{2} q-\left(\alpha_{0}+\alpha_{2}\right) t\right)\left(p q(q-t)+\left(\alpha_{1}+\alpha_{2}\right) q-\left(\alpha_{0}+\alpha_{1}+\alpha_{2}\right) t\right)} \\
& T_{\omega_{3}}(p)=-\frac{\left(p q(q-t)+\alpha_{2} q-\left(\alpha_{0}+\alpha_{2}\right) t\right)\left(p q(q-t)+\left(\alpha_{1}+\alpha_{2}\right) q-\left(\alpha_{0}+\alpha_{1}+\alpha_{2}\right) t\right)}{t\left(p(q-t)-\alpha_{0}\right)} \\
& \cdot \frac{\left(p^{2} q(q-t)+p\left(\alpha_{1}+2 \alpha_{2}\right) q-\left(\alpha_{0}+\alpha_{1}+2 \alpha_{2}\right) t+\alpha_{2}\left(\alpha_{1}+\alpha_{2}\right)\right)}{\left(p^{2} q(q-t)^{2}+p(q-t)\left(\left(\alpha_{1}+2 \alpha_{2}\right) q+\alpha_{4} t\right)+\alpha_{2}\left(\alpha_{1}+\alpha_{2}\right) q-\left(\left(\alpha_{0}+\alpha_{2}\right)^{2}+\left(\alpha_{0} \alpha_{1}+\alpha_{2} \alpha_{1}+\alpha_{0} \alpha_{4}\right)\right) t\right)},
\end{aligned}
$$

and then substitute the seed solution $(q, p)=\left(x, \frac{1}{2}\left(a+b-\frac{1}{2}\right) x^{-1}\right), x=t^{\frac{1}{2}}$, with parameters $\alpha=\left(a, b, \frac{1}{2}-a-b, a, b\right)$ to obtain an algebraic solution

$$
\begin{aligned}
& q_{1}=\frac{x\left(\left(a-b-\frac{1}{2}\right) x+a+b+\frac{1}{2}\right)\left(\left(a+b-\frac{1}{2}\right) x+a-b+\frac{1}{2}\right)}{\left(\left(a-b+\frac{1}{2}\right) x+a+b-\frac{1}{2}\right)\left(\left(a+2 b+\frac{1}{2}\right) x+a-b-\frac{1}{2}\right)} \\
& p_{1}=\frac{\left(a+b-\frac{1}{2}\right)\left(\left(a-b+\frac{1}{2}\right) x+a+b-\frac{1}{2}\right)\left(\left(a+b-\frac{3}{2}\right) x+a-b-\frac{1}{2}\right)\left(\left(a+b+\frac{1}{2}\right) x+a-b-\frac{1}{2}\right)}{\left.2 x\left(\left(a+b-\frac{1}{2}\right) x+a-b+\frac{1}{2}\right)\left(\left(a-\frac{1}{2}\right)^{2}-b^{2}\right) x^{2}+2\left(\left(a-\frac{1}{2}\right)^{2}+b^{2}-\frac{1}{2}\right) x+\left(a-\frac{1}{2}\right)^{2}-b^{2}\right)}
\end{aligned}
$$

with parameters $\left(a+1, b, \frac{1}{2}-a-b, a-1, b\right)$. In this example, one can already observe a characteristic phenomenon of factorization of the action of $\widetilde{W}$. The Umemura polynomials arise as the special polynomials in $x=t^{\frac{1}{2}}$ associated with the sequence of $\tau$ functions for these algebraic solutions with parameters $\left(a+m, b, \frac{1}{2}-a-b, a-m, b\right)(m \in \mathbb{Z})$.

We now consider to extend the action of $\widetilde{W}=\left\langle s_{0}, s_{1}, s_{2}, s_{3}, s_{4} ; r_{1}, r_{3}, r_{4}\right\rangle$ on $\mathcal{K}=\mathbb{C}(\alpha ; q, p, t)$ to the level of $\tau$ functions. We introduce four $\tau$ functions $\tau_{0}, \tau_{1}, \ldots, \tau_{4}$ corresponding to the four nodes of the Dynkin diagram (more precisely, they are multiplicative variables attached to the affine fundamental weights $\left.\Lambda_{0}, \Lambda_{1}, \ldots, \Lambda_{4}\right)$. We need to specify the differential equations to be satisfied by $\tau_{i}$ as well as how they should behave under the action of $\widetilde{W}$. From the viewpoint of Weyl group actions of Noumi-Yamada [11], it is natural to 
assume that the simple reflections $s_{i}(i=0,1, \ldots, 4)$ act on the $\tau$ functions $\tau_{j}(j=0,1, \ldots, 4)$ as

$$
\begin{gathered}
s_{i}\left(\tau_{j}\right)=\tau_{j} \quad(i, j \in\{0,1,2,3,4\} ; j \neq i) ; \quad s_{0}\left(\tau_{0}\right)=c_{0}(t)(q-t) \frac{\tau_{2}}{\tau_{0}}, \\
s_{1}\left(\tau_{1}\right)=c_{1}(t) \frac{\tau_{2}}{\tau_{1}}, \quad s_{2}\left(\tau_{2}\right)=c_{2}(t) p \frac{\tau_{0} \tau_{1} \tau_{3} \tau_{4}}{\tau_{2}}, \\
s_{3}\left(\tau_{3}\right)=c_{3}(t)(q-1) \frac{\tau_{0}}{\tau_{3}}, \quad s_{4}\left(\tau_{4}\right)=c_{4}(t) \frac{\tau_{2}}{\tau_{4}}
\end{gathered}
$$

with some nonzero functions $c_{i}(t)$ of $t$ which do not depend on $\alpha$ parameters. The factors $f_{0}=q-t, f_{1}=1, f_{2}=p, f_{3}=q-1, f_{4}=q$ are the "invariant divisors" chosen as the generators of the Poisson algebra $\mathbb{C}[q, p, t]$ that are subject to the Serre relations of type $D_{4}^{(1)}$ with respect to Poisson adjoint actions. (The $\tau$ factors on the right-hand sides come from the action of simple affine reflections $s_{i}$ on the affine fundamental weights $\Lambda_{j}$.) Also, the differential equations for $\tau_{i}$ should be expressed as

$$
t(t-1) \partial_{t}\left(\tau_{i}\right)=h_{i} \tau_{i}, \quad \text { namely, } h_{i}=\frac{t(t-1) \partial_{t}\left(\tau_{i}\right)}{\tau_{i}} \quad(i=0,1, \ldots, 4)
$$

with appropriate Hamiltonian functions $h_{0}, h_{1}, \ldots, h_{4} \in \mathbb{C}(\alpha)[q, p, t]$. Although the choice of $c_{i}(t)$ and $h_{0}, h_{1}, \ldots, h_{4}$ is not unique, in this paper we simply follow the convention of Tetsu Masuda [8], where $c_{i}(t)=1$ $(i=0,1,3,4), c_{2}(t)=t^{-\frac{1}{2}}$; we do no specify $h_{0}, h_{1}, \ldots, h_{4}$ here, and refer to [8] for their definitions. Also, the action of $r_{1}, r_{3}, r_{4}$ can be extended in the form

$$
\begin{aligned}
& r_{k}\left(\tau_{i}\right)=d_{k, i}(t) \tau_{\sigma_{k}(i)} \quad(i=0,1,3,4) ; \\
& r_{1}\left(\tau_{2}\right)=e_{1}(t)(q-t) \tau_{2}, \quad r_{3}\left(\tau_{2}\right)=e_{3}(t)(q-1) \tau_{2}, \quad r_{4}\left(\tau_{2}\right)=e_{4}(t) q \tau_{2}
\end{aligned}
$$

with some functions $c_{k, i}(t), d_{k}(t) \in \mathbb{C}\left(t^{\frac{1}{4}},(t-1)^{\frac{1}{4}}\right)$; we do not specify these functions since they depend on the convention. (In [8], $r_{1}, r_{3}, r_{4}$ are denoted by $s_{5}, s_{6}, s_{7}$ respectively.) The derivation $\partial_{t}$ on $\mathcal{K}=\mathbb{C}(\alpha ; q, p, t)$ is extended in this way to the ring of Laurent polynomials $\mathcal{L}=\widetilde{\mathcal{K}}\left[\tau^{ \pm 1}\right]=$ $\widetilde{\mathcal{K}}\left[\tau_{0}^{ \pm 1}, \tau_{1}^{ \pm 1}, \ldots, \tau_{4}^{ \pm 1}\right]$, where $\widetilde{\mathcal{K}}=\mathbb{C}\left(\alpha ; q, p, t, t^{\frac{1}{4}},(t-1)^{\frac{1}{4}}\right)$. Also, the automorphisms $s_{0}, s_{1}, \ldots, s_{4}, r_{1}, r_{3}, r_{4}$ on $\mathcal{K}$ are extended to $\mathcal{L}=\widetilde{\mathcal{K}}\left[\tau^{ \pm 1}\right]$ so that they commute with $\partial_{t}$. The automorphisms $s_{0}, s_{1}, s_{2}, s_{3}, s_{4}: \mathcal{L} \rightarrow \mathcal{L}$ satisfy the fundamental relations of the affine Weyl group $W=\left\langle s_{0}, s_{1}, s_{2}, s_{3}, s_{4}\right\rangle$. However, it should be noted that $r_{1}, r_{3}, r_{4}: \mathcal{L} \rightarrow \mathcal{L}$ do not satisfy all the fundamental relations of the Dynkin diagram automorphisms when they act on $\tau$ functions. We denote by $\widehat{W}$ the subgroup of $\operatorname{Aut}(\mathcal{L})$ generated by $s_{i}$ and $r_{i}$. Then $\widehat{W}$ is a bigger group than $\widetilde{W}$, and $\widetilde{W}$ is a quotient of $\widehat{W}$. It is a subtle question whether or not one can preserve the whole set of fundamental relations of the extended affine Weyl group $\widetilde{W}$, in lifting its action on $\mathcal{K}$ to the level of $\tau$ functions. 
We take the lift

$$
\widehat{T}_{\omega_{3}}=r_{3} s_{3} s_{2} s_{1} s_{4} s_{2} s_{3}: \widetilde{\mathcal{K}}\left[\tau^{ \pm 1}\right] \rightarrow \widetilde{\mathcal{K}}\left[\tau^{ \pm 1}\right]
$$

of the translation $T_{\omega_{3}}: \mathcal{K} \rightarrow \mathcal{K}$, and consider the sequence of $\tau$ functions $\tau^{(m)}=\widehat{T}_{\omega_{3}}^{m}\left(\tau_{0}\right)(m \in \mathbb{Z})$. By the action of $\widehat{W}$ on $\mathcal{L}=\widetilde{\mathcal{K}}\left[\tau^{ \pm 1}\right]$, one can directly verify

$$
t^{\frac{1}{2}} \frac{\widehat{T}_{\omega_{3}}(\tau) \widehat{T}_{\omega_{3}}^{-1}\left(\tau_{0}\right)}{\tau_{0}^{2}}=(t-1)^{2} \partial_{t}\left(\frac{t \partial_{t}(\tau)}{\tau}\right)+\frac{1}{4}\left(\alpha_{0}-\alpha_{3}-1\right)^{2}+\frac{1}{2}
$$

Applying $\widehat{T}_{\omega_{3}}^{m}$ to this equality, we obtain the Toda equation

$t^{\frac{1}{2}} \frac{\tau^{(m+1)} \tau^{(m-1)}}{\left(\tau^{(m)}\right)^{2}}=(t-1)^{2} \partial_{t}\left(\frac{t \partial_{t}\left(\tau^{(m)}\right)}{\tau^{(m)}}\right)+\frac{1}{4}\left(\alpha_{0}-\alpha_{3}+2 m-1\right)^{2}+\frac{1}{2} \quad(m \in \mathbb{Z})$

for the sequence of $\tau$ functions $\tau^{(m)}=\widehat{T}_{\omega_{3}}^{m}\left(\tau_{0}\right)$ with parameter values

$$
\left(T_{\omega_{3}}^{m}\left(\alpha_{0}\right), \ldots, T_{\omega_{3}}^{m}\left(\alpha_{4}\right)\right)=\left(\alpha_{0}+m, \alpha_{1}, \alpha_{2}, \alpha_{3}-m, \alpha_{4}\right) .
$$

We specialize this Toda equation to the seed solution

$$
\begin{aligned}
(q, p)=\left(t^{\frac{1}{2}}, \frac{1}{2}\right. & \left.\left(a+b-\frac{1}{2}\right) t^{-\frac{1}{2}}\right) \\
& \text { with }\left(\alpha_{0}, \alpha_{1}, \alpha_{2}, \alpha_{3}, \alpha_{4}\right)=\left(a, b, \frac{1}{2}-a-b, a, b\right)
\end{aligned}
$$

at the fixed point of $r_{3}$. Note that, for this seed solution, $\tau_{0}, \tau_{3}$ can be taken as

$$
\tau_{0}=t^{-\frac{1}{2}\left(\left(a-\frac{1}{2}\right)^{2}+b^{2}+\frac{1}{8}\right)}\left(t^{\frac{1}{2}}-1\right)^{\left(a-\frac{1}{2}\right)^{2}+\frac{1}{2}}\left(t^{\frac{1}{2}}+1\right)^{b^{2}+\frac{1}{2}}, \quad \tau_{3}=t^{-\frac{1}{4}} \tau_{0} .
$$

By this specialization, we obtain the Toda equation

$$
t^{\frac{1}{2}} \frac{\tau^{(m+1)} \tau^{(m-1)}}{\left(\tau^{(m)}\right)^{2}}=(t-1)^{2} \partial_{t}\left(\frac{t \partial_{t}\left(\tau^{(m)}\right)}{\tau^{(m)}}\right)+\frac{1}{4}(2 m-1)^{2}+\frac{1}{2} \quad(m \in \mathbb{Z})
$$

for the $\tau$ functions $\tau^{(m)}$ of the algebraic solutions with parameters $(a+m$, $\left.b, \frac{1}{2}-a-b, a-m, b\right)$. Furthermore, by setting $\tau^{(m)}=\phi_{m} \tau_{0}^{1-m} \tau_{3}^{m} t^{\frac{1}{4}\left(3 m-2 m^{2}\right)}$, we obtain the recurrence formula for $\phi_{m}=\phi_{m}(x), x=t^{\frac{1}{2}}$ :

$$
\begin{aligned}
4 \phi_{m+1} \phi_{m-1}= & \left(x^{2}-1\right)^{2}\left(x \phi_{m}^{\prime \prime} \phi_{m}+\phi_{m}^{\prime} \phi_{m}-x\left(\phi_{m}^{\prime}\right)^{2}\right) \\
& -\left(\left(a-\frac{1}{2}\right)^{2}(x+1)^{2}-b^{2}(x-1)^{2}-(2 m-1)^{2} x\right) \phi_{m}^{2},
\end{aligned}
$$

where $^{\prime}=\mathrm{d} / \mathrm{d} x$. Noting that $\phi_{0}, \phi_{1} \in \mathbb{C}$, we set $\phi_{m}=c_{m} U_{m}$ with the constants $c_{m}(m \in \mathbb{Z})$ determined by

$$
c_{0}=\phi_{0}, \quad c_{1}=\phi_{1}, \quad 4 c_{m+1} c_{m-1}=c_{m}^{2} \quad(m \in \mathbb{Z}) .
$$


Then the Toda equation for $U_{m}=U_{m}(x)$ is expressed as

$$
\begin{aligned}
U_{m+1} U_{m-1}= & \left(x^{2}-1\right)^{2}\left(x U_{m}^{\prime \prime} U_{m}+U_{m}^{\prime} U_{m}-x\left(U_{m}^{\prime}\right)^{2}\right) \\
& -\left(\left(a-\frac{1}{2}\right)^{2}(x+1)^{2}-b^{2}(x-1)^{2}-(2 m-1)^{2} x\right) U_{m}^{2} \quad(m \in \mathbb{Z}),
\end{aligned}
$$

with the initial condition $U_{0}=U_{1}=1$. This is the recurrence formula for the Umemura polynomials $U_{m}$ that we discussed in Section 1 with $a$ replaced by $a-\frac{1}{2}$.

Following Masuda [8], we make use of two translations $T_{\varepsilon_{4}}, T_{-\varepsilon_{3}}$ in order to generate a two-parameter family of algebraic solutions from the seed solution

$$
(q, p)=\left(x, \frac{1}{2}\left(a+b-\frac{1}{2}\right) x^{-1}\right), \quad\left(\alpha_{0}, \alpha_{1}, \alpha_{2}, \alpha_{3}, \alpha_{4}\right)=\left(a, b, \frac{1}{2}-a-b, a, b\right)
$$

at the fixed point of $r_{3}$, where $x=t^{\frac{1}{2}}$. The translations $T_{\varepsilon_{4}}$ and $T_{-\varepsilon_{3}}$ act on the parameter as

\begin{tabular}{|c||ccccc|}
\hline & $\alpha_{0}$ & $\alpha_{1}$ & $\alpha_{2}$ & $\alpha_{3}$ & $\alpha_{4}$ \\
\hline \hline$T_{\varepsilon_{4}}$ & $\alpha_{0}$ & $\alpha_{1}$ & $\alpha_{2}$ & $\alpha_{3}+1$ & $\alpha_{4}-1$ \\
$T_{-\varepsilon_{3}}$ & $\alpha_{0}$ & $\alpha_{1}$ & $\alpha_{2}-1$ & $\alpha_{3}+1$ & $\alpha_{4}+1$ \\
\hline
\end{tabular}

Hence, for each $(m, n) \in \mathbb{Z}^{2}$, by the Bäcklund transformation

$$
q_{m, n}=T_{\varepsilon_{4}}^{m} T_{-\varepsilon_{3}}^{n}(q), \quad p_{m, n}=T_{\varepsilon_{4}}^{m} T_{-\varepsilon_{3}}^{n}(p)
$$

we obtain an algebraic solution $\left(q_{m, n}, p_{m, n}\right)$ with parameter values

$$
\left(a, b, \frac{1}{2}-a-b-n, a+m+n, b-m+n\right) .
$$

In order to define $\tau$ functions for this family, we use the lifts

$$
\widehat{T}_{\varepsilon_{4}}=s_{3} s_{2} s_{0} s_{1} s_{2} s_{3} r_{1}, \quad \widehat{T}_{-\varepsilon_{3}}=s_{3} s_{4} s_{2} s_{0} s_{1} s_{2} r_{1}: \quad \widetilde{\mathcal{K}}\left[\tau^{ \pm 1}\right] \rightarrow \widetilde{\mathcal{K}}\left[\tau^{ \pm 1}\right],
$$

and set

$$
\tau_{m, n}=\widehat{T}_{\varepsilon_{4}}^{m} \widehat{T}_{-\varepsilon_{3}}^{n}\left(\tau_{0}\right) \quad(m, n \in \mathbb{Z}) .
$$

It is known by Masuda [8] that, when specialized to the seed solution (3.25), these $\tau$ functions are expressed in the form

$$
\tau_{m, n}=c_{m, n} x^{\lambda_{m, n}}(x+1)^{\mu_{m, n}}(x-1)^{\nu_{m, n}} V_{m, n}(x ; a, b),
$$

where $c_{m, n}, \lambda_{m, n}, \mu_{m, n}, \nu_{m, n}$ are constants determined by $a, b$ and $m, n$, and $V_{m, n}(x ; a, b)$ is a polynomial in $x$ with parameters $(a, b)$. These polynomials $V_{m, n}(x ; a, b)$ are by definition the Umemura polynomials with two discrete parameters $m, n$ for this family of algebraic solutions. Furthermore, 
the algebraic solution $\left(q_{m, n}, p_{m, n}\right)$ with parameter values $\left(a, b, \frac{1}{2}-a-b-n\right.$, $a+m+n, b-m+n)$ is expressed as

$$
\begin{aligned}
q_{m, n}= & x \frac{V_{m, n-1}(x ; a+1, b) V_{m-1, n}(x ; a+1, b)}{V_{m-1, n-}(x ; a+1, b+1) V_{m, n-1}(x ; a+1, b+1)} \\
p_{m, n}= & \frac{1}{2}\left(a+b+n-\frac{1}{2}\right) \\
& \cdot x^{-1} \frac{V_{m-1, n}(x ; a+1, b-1) V_{m, n-1}(x ; a+1, b+1) V_{m, n-1}(x ; a, b)}{V_{m, n}(x ; a, b) V_{m-1, n-1}(x ; a+1, b) V_{m, n-1}(x ; a+1, b)},
\end{aligned}
$$

for each $m, n \in \mathbb{Z}$. Also, these polynomials $V_{m, n}=V_{m, n}(x ; a, b)(m, n \in \mathbb{Z})$ are characterized by the Toda equations

$$
\begin{aligned}
& \frac{1}{2}\left(a-b+m-\frac{1}{2}\right) V_{m+1, n} V_{m-1, n} \\
& =x\left(x^{2}-1\right)\left(\ddot{V}_{m, n} V_{m, n}-\dot{V}_{m, n}^{2}\right)+\left(3 x^{2}-1\right) \dot{V}_{m, n} V_{m, n} \\
& \quad+\left\{\left(a-b+m-\frac{1}{2}\right)\left[\left(a-b+3 m+\frac{1}{2}\right) x+\left(a+b+n-\frac{1}{2}\right)\right]-n(n+1) x\right\} V_{m, n}^{2} \\
& \frac{1}{2}\left(a+b+n-\frac{1}{2}\right) V_{m, n+1} V_{m, n-1} \\
& =x\left(x^{2}-1\right)\left(\ddot{V}_{m, n} V_{m, n}-\dot{V}_{m, n}^{2}\right)+\left(3 x^{2}-1\right) \dot{V}_{m, n} V_{m, n} \\
& \quad+\left\{\left(a+b+n-\frac{1}{2}\right)\left[\left(a+b+3 n+\frac{1}{2}\right) x+\left(a-b+m-\frac{1}{2}\right)\right]-m(m+1) x\right\} V_{m, n}^{2},
\end{aligned}
$$

with initial conditions

$$
V_{-1,-1}=V_{-1,0}=V_{0,-1}=V_{0,0}=1,
$$

where $\dot{V}_{m, n}=\mathrm{d} V_{m, n} / \mathrm{d} x$ and $\ddot{V}_{m, n}=\mathrm{d}^{2} V_{m, n} / \mathrm{d} x^{2}([8$, Proposition 4.4]). For other characterization of these polynomials see [8]. One of the main results of Masuda [8] is an explicit determinant formula for $V_{m, n}(x ; a, b)$ (as a universal character) in terms of certain Jacobi polynomials, but we will not get into this topic here.

We remark that this family of Umemura polynomials $V_{m, n}(x ; a, b)$ satisfies

$$
V_{m, n}(x ; a, b)=V_{n, m}(x ; a,-b) \quad(m, n \in \mathbb{Z}) .
$$

From the combinatorial point of view, it seems appropriate to reparametrize these polynomials as

$$
\begin{aligned}
U_{m, n}(x ; c, d)= & V_{m, n}(x ; a, b) \\
& \text { with } \begin{cases}c=2 a+m+n-1, & d=2 b-m+n, \\
a=\frac{1}{2}(c-m-n+1), & b=\frac{1}{2}(d+m-n) .\end{cases}
\end{aligned}
$$


Then one can show that

$$
\begin{aligned}
& U_{m, n}(x ; c, d)=U_{n, m}(x ; c,-d), \\
& U_{m, n}(x ; c, d)=(-1)^{\left(\begin{array}{c}
m+1 \\
2
\end{array}\right)+\left(\begin{array}{c}
n+1 \\
2
\end{array}\right)} U_{m, n}(x ;-c,-d),
\end{aligned}
$$

and

$$
\begin{aligned}
& U_{m, n}(x ; c, d)=U_{-1-m, n}(x ; c, d) \\
& \quad=U_{m,-1-n}(x ; c, d)=U_{-1-m,-1-n}(x ; c, d) .
\end{aligned}
$$

Through the parameterization (3.36), these polynomials $U_{m, n}(x ; c, d)$ correspond to the parameter values

$$
\begin{aligned}
& \frac{1}{2}(c+1-m-n, d+m-n,-c-d, c+1+m+n, d-m+n) \\
& =\frac{1}{2}(c+1, d,-c-d, c+1, d)+\frac{m}{2}(-1,1,0,1,-1)+\frac{n}{2}(-1,-1,0,1,1) .
\end{aligned}
$$

In particular, the subfamily $U_{-m,-m}(x ; c, d)$ correspond to algebraic solutions with parameters

$$
\left(\frac{1}{2}(c+1)+m, \frac{1}{2} d,-\frac{1}{2}(c+d), \frac{1}{2}(c+1)-m, \frac{1}{2} d\right) .
$$

We remark that $U_{-m,-m}(x ; c, d)$ coincides with a constant multiple of the Umemura polynomial $U_{m}(x ; c, d)$ that we discussed before in (3.24) with $a-\frac{1}{2}=\frac{1}{2} c, b=\frac{1}{2} d$.

In the next section, we formulate a conjecture on the combinatorial formula for this family $U_{m, n}(x ; c, d)$ of special polynomials.

Remark 3.1. - There are many related subjects that we could not cover here. The classification of algebraic solutions of $P_{\mathrm{VI}}$ has been completed by contribution of many researchers (see Lisovyy-Tykhyy [6] and references therein). Also, interesting developments are going on in understanding the $\tau$ functions for general solutions of Painlevé equations in connection with the conformal field theory. It would also be an intriguing problem to study special polynomials of Umemura type for the discrete Painlevé equations in Sakai's list.

\section{Combinatorial Umemura polynomials: a conjecture}

In this section we introduce a class of combinatorial polynomials $G_{\Lambda}(u, v ; \boldsymbol{c}, \boldsymbol{d})$, which we call the combinatorial Umemura polynomials. We also formulate a conjecture of combinatorial formula for Umemura polynomials $U_{m, n}(x ; c, d)$ with two discrete parameters, in terms of these combinatorial polynomials. This part is based on the collaboration of the author with 
Hiroshi Kageyama and Takuya Yoshida who were students in the master's course of Kobe University.

Let $\boldsymbol{c}=\left(c_{1}, c_{2}, \ldots\right)$ and $\boldsymbol{d}=\left(d_{1}, d_{2}, \ldots\right)$ be two sequences of parameters. For each finite set $\Lambda=\left\{l_{1}, \ldots, l_{n}\right\}$ of positive integers with $|\Lambda|=n$, we define a polynomial $G_{\Lambda}(u, v ; \boldsymbol{c}, \boldsymbol{d})$ in two variables $(u, v)$ by

$$
\begin{aligned}
& G_{\Lambda}(u, v ; \boldsymbol{c}, \boldsymbol{d}) \\
& =\sum_{I \sqcup J=\Lambda}(-1)^{\left(\begin{array}{c}
|I| \\
2
\end{array}\right)+\sum_{i \in I}(i-1)} \prod_{i \in I ; j \in J} \frac{j+i}{j-i} \prod_{i \in I} c_{i} u^{i} \prod_{j \in J} d_{j} v^{j} \\
& =\sum_{I \sqcup J=\{1, \ldots, n\}}(-1)^{\left(\begin{array}{c}
|I| \\
2
\end{array}\right)+\sum_{i \in I}\left(l_{i}-1\right)} \prod_{i \in I ; j \in J} \frac{l_{j}+l_{i}}{l_{j}-l_{i}} \prod_{i \in I} c_{l_{i}} u^{l_{i}} \prod_{j \in J} d_{l_{j}} v^{l_{j}} .
\end{aligned}
$$

We call $G_{\Lambda}(u, v ; \boldsymbol{c}, \boldsymbol{d})$ the combinatorial Umemura polynomial attached to $\Lambda$. Note that $\Lambda$ can be identified with a strict partition $\lambda=\left(\lambda_{1}, \ldots, \lambda_{n}\right)$, $\lambda_{1}>\cdots>\lambda_{n}$, by rearranging the elements of $\Lambda$ in the decreasing order. As a polynomial is $(u, v), G_{\Lambda}(u, v ; \boldsymbol{c}, \boldsymbol{d})$ is homogenous of degree $|\lambda|=\lambda_{1}+\cdots+\lambda_{n}$.

Besides the combinatorial formula (4.1), two determinant formulas are known for $G_{\Lambda}(u, v ; \boldsymbol{c}, \boldsymbol{d})$; one of Vandermonde type, due to Yoshida [21], and the other of Cauchy type, due to Kirillov-Taneda [5] and Kageyama [3].

TheOREM 4.1 (Yoshida [21]). — For any finite set $\Lambda=\left\{l_{1}, l_{2}, \ldots, l_{n}\right\}$ of positive integers with $|\Lambda|=n$, we have

$$
G_{\Lambda}(u, v ; \boldsymbol{c}, \boldsymbol{d})=\frac{1}{\Delta\left(l_{1}, \ldots, l_{n}\right)} \operatorname{det}\left(l_{j}^{n-i}\left((-1)^{n-i+l_{j}-1} c_{l_{j}} u^{l_{j}}+d_{l_{j}} v^{l_{j}}\right)\right)_{i, j=1}^{n},
$$

where $\Delta\left(l_{1}, \ldots, l_{n}\right)=\prod_{1 \leqslant i<j \leqslant n}\left(l_{i}-l_{j}\right)$ stands for the difference product.

This theorem follows from the following determinant formula of rational functions in the variables $\left(x_{1}, \ldots, x_{n}\right)$ with parameters $\left(\alpha_{1}, \ldots, \alpha_{n}\right)$, $\left(\beta_{1}, \ldots, \beta_{n}\right)$ and $t$.

LEMMA 4.2. -

$$
\begin{aligned}
\sum_{I \sqcup J=\{1, \ldots, n\}} t^{\left(\begin{array}{c}
|I| \\
2
\end{array}\right)} \prod_{i \in I ; j \in J} \frac{t x_{i}-x_{j}}{x_{i}-x_{j}} \prod_{i \in I} \alpha_{i} \prod_{j \in J} \beta_{j} \\
=\frac{\operatorname{det}\left(x_{j}^{n-i}\left(\beta_{j}+t^{n-i} \alpha_{j}\right)\right)_{i, j=1}^{n}}{\Delta\left(x_{1}, \ldots, x_{n}\right)}
\end{aligned}
$$

In fact, formula (4.2) is a special case of (4.3) where $t=-1$ and

$$
x_{j}=l_{j}, \quad \alpha_{j}=(-1)^{l_{j}-1} c_{l_{j}} u^{l_{j}}, \quad \beta_{j}=d_{l_{j}} v^{l_{j}} \quad(j=1, \ldots, n) .
$$


Proof of Lemma 4.2. - Note first that

$$
\frac{\prod_{i \in I} T_{t, x_{i}} \Delta\left(x_{1}, \ldots, x_{n}\right)}{\Delta\left(x_{1}, \ldots, x_{n}\right)}=t^{\left(\begin{array}{l}
|I| \\
2
\end{array}\right)} \prod_{i \in I ; j \notin I} \frac{t x_{i}-x_{j}}{x_{i}-x_{j}}
$$

for any subset $I \subseteq\{1, \ldots, n\}$. Here, for each $i=1, \ldots, n, T_{t, x_{i}}$ stands for the multiplicative shift operator in $x_{i}$ by $t: T_{t, x_{i}} f\left(x_{1}, \ldots, x_{i}, \ldots, x_{n}\right)=$ $f\left(x_{1}, \ldots, t x_{i}, \ldots, x_{n}\right)$. Hence we have

$$
\begin{gathered}
\sum_{I \sqcup J=\{1, \ldots, n\}} t^{\left(\begin{array}{l}
|I| \\
2
\end{array}\right)} \prod_{i \in I ; j \in J} \frac{t x_{i}-x_{j}}{x_{i}-x_{j}} \prod_{i \in I} \alpha_{i} \prod_{j \in J} \beta_{j} \\
=\sum_{I \sqcup J=\{1, \ldots, n\}} \prod_{j \in j} \beta_{j} \prod_{i \in I} \alpha_{i} \frac{\prod_{i \in I} T_{t, x}^{I} \Delta\left(x_{1}, \ldots, x_{n}\right)}{\Delta\left(x_{1}, \ldots, x_{n}\right)} \\
=\frac{1}{\Delta\left(x_{1}, \ldots, x_{n}\right)}\left(\sum_{I \sqcup J=\{1, \ldots, n\}} \prod_{j \in J} \beta_{j} \prod_{i \in I} \alpha_{i} T_{t, x_{i}}\right) \Delta\left(x_{1}, \ldots, x_{n}\right) \\
=\frac{1}{\Delta\left(x_{1}, \ldots, x_{n}\right)} \prod_{k=1}^{n}\left(\beta_{k}+\alpha_{k} T_{t, x_{k}}\right) \Delta\left(x_{1}, \ldots, x_{n}\right) .
\end{gathered}
$$

By $\Delta\left(x_{1}, \ldots, x_{n}\right)=\operatorname{det}\left(x_{j}^{n-i}\right)_{i, j=1}^{n}$, we obtain

$$
\begin{aligned}
\sum_{I \sqcup J=\{1, \ldots, n\}} t^{\left(\begin{array}{l}
|I| \\
2
\end{array}\right)} & \prod_{i \in I ; j \in J} \frac{t x_{i}-x_{j}}{x_{i}-x_{j}} \prod_{i \in I} \alpha_{i} \prod_{j \in J} \beta_{j} \\
& =\frac{1}{\Delta\left(x_{1}, \ldots, x_{n}\right)} \prod_{k=1}^{n}\left(\beta_{k}+\alpha_{k} T_{t, x_{k}}\right) \operatorname{det}\left(x_{j}^{n-i}\right)_{i, j=1}^{n} \\
& =\frac{1}{\Delta\left(x_{1}, \ldots, x_{n}\right)} \operatorname{det}\left(\left(\beta_{j}+\alpha_{j} T_{t, x_{j}}\right) x_{j}^{n-i}\right)_{i, j=1}^{n} \\
& =\frac{1}{\Delta\left(x_{1}, \ldots, x_{n}\right)} \operatorname{det}\left(x_{j}^{n-i}\left(\beta_{j}+t^{n-i} \alpha_{j}\right)\right)_{i, j=1}^{n} .
\end{aligned}
$$

Theorem 4.3 (Kirillov-Taneda [5], Kageyama [3]). — For any finite set $\Lambda=\left\{l_{1}, l_{2}, \ldots, l_{n}\right\}$ of positive integers with $|\Lambda|=n$, we have

$$
\begin{aligned}
& G_{\Lambda}(u, v ; \boldsymbol{c}, \boldsymbol{d}) \\
& \quad=\operatorname{det}\left(d_{i} v^{i} \delta_{i, j}+(-1)^{l_{i}-1} c_{l_{i}} u^{l_{i}} \frac{2 l_{i}}{l_{i}+l_{j}} \prod_{1 \leqslant k \leqslant n ; k \neq i} \frac{l_{k}+l_{i}}{l_{k}-l_{i}}\right)_{i, j=1}^{n} .
\end{aligned}
$$

This theorem follows from the following determinant formula of rational functions in the variables $\left(x_{1}, \ldots, x_{n}\right)$ and parameters $\left(\alpha_{1}, \ldots, \alpha_{n}\right)$, $\left(\beta_{1}, \ldots, \beta_{n}\right)$. 
LEMMA 4.4. -

$$
\begin{aligned}
\sum_{I \sqcup J=\{1, \ldots, n\}}(-1)^{\left(\begin{array}{c}
|I| \\
2
\end{array}\right)} & \prod_{\substack{i \in I \\
j \in J}} \frac{1-x_{i} x_{j}}{x_{i}-x_{j}} \prod_{i \in I} \alpha_{i} \prod_{j \in J} \beta_{j} \\
& =\operatorname{det}\left(\beta_{i} \delta_{i, j}+\alpha_{i} \frac{1-x_{i}^{2}}{1-x_{i} x_{j}} \prod_{\substack{1 \leqslant k \leqslant n \\
k \neq i}} \frac{1-x_{i} x_{k}}{x_{i}-x_{k}}\right)_{i, j=1}^{n}
\end{aligned}
$$

In this formula, set $x_{k}=1-q^{l_{k}}(k=1, \ldots, n)$ and take the limit as $q \rightarrow 1$ to obtain

$$
\begin{gathered}
\sum_{I \sqcup J=\{1, \ldots, n\}}(-1)^{\left(\begin{array}{c}
|I| \\
2
\end{array}\right)} \prod_{i \in I} \alpha_{i} \prod_{j \in J} \beta_{j} \prod_{i \in I ; j \in J} \frac{l_{j}+l_{i}}{l_{j}-l_{i}} \\
=\operatorname{det}\left(\beta_{i} \delta_{i, j}+\alpha_{i} \frac{2 l_{i}}{l_{i}+l_{j}} \prod_{\substack{1 \leqslant k \leqslant n \\
j \neq i}} \frac{l_{k}+l_{i}}{l_{k}-l_{i}}\right)_{i, j=1}^{n} .
\end{gathered}
$$

Setting

$$
\alpha_{i}=(-1)^{l_{i}-1} c_{l_{i}} u^{l_{i}}, \quad \beta_{i}=d_{l_{i}} v^{l_{i}} \quad(i=1, \ldots, n),
$$

we obtain

$$
\begin{aligned}
& \sum_{I \sqcup J=\{1, \ldots, n\}}(-1)^{\left(\begin{array}{c}
|I| \\
2
\end{array}\right)+\sum_{i \in I}\left(l_{i}-1\right)} \prod_{i \in I} c_{l_{i}} u^{l_{i}} \prod_{j \in J} d_{l_{j}} v^{l_{j}} \prod_{i \in I ; j \in J} \frac{l_{j}+l_{i}}{l_{j}-l_{i}} \\
= & \operatorname{det}\left(d_{l_{i}} v^{l_{i}} \delta_{i, j}+(-1)^{l_{i}-1} c_{l_{i}} u^{l_{i}} \frac{2 l_{i}}{l_{i}+l_{j}} \prod_{1 \leqslant k \leqslant n ; k \neq i} \frac{l_{k}+l_{i}}{l_{k}-l_{i}}\right)_{i, j=1}^{n} .
\end{aligned}
$$

Proof of Lemma 4.4. - In Cauchy's determinant formula

$$
\operatorname{det}\left(\frac{1}{1-x_{i} y_{j}}\right)_{i, j=1}^{n}=\frac{\Delta\left(x_{1}, \ldots, x_{n}\right) \Delta\left(y_{1}, \ldots, y_{n}\right)}{\prod_{i, j=1}^{n}\left(1-x_{i} y_{j}\right)},
$$

multiply each row by $1-x_{i}^{2}$ for $i=1, \ldots, n$ to obtain

$$
\operatorname{det}\left(\frac{1-x_{i}^{2}}{1-x_{i} y_{j}}\right)_{i, j=1}^{n}=\prod_{i=1}^{n}\left(1-x^{2}\right) \frac{\Delta\left(x_{1}, \ldots, x_{n}\right) \Delta\left(y_{1}, \ldots, y_{n}\right)}{\prod_{i, j=1}^{n}\left(1-x_{i} y_{j}\right)}
$$

Setting $y_{j}=x_{j}(j=1, \ldots, n)$ we obtain

$$
\operatorname{det}\left(\frac{1-x_{i}^{2}}{1-x_{i} x_{j}}\right)_{i, j=1}^{n}=(-1)^{\left(\begin{array}{c}
n \\
2
\end{array}\right)} \prod_{1 \leqslant i, j \leqslant n ; i \neq j} \frac{x_{i}-x_{j}}{1-x_{i} x_{j}} .
$$


When $I \sqcup J=\{1, \ldots, n\}$,

$$
\begin{aligned}
\prod_{i \in I ; j \in J} \frac{1-x_{i} x_{j}}{x_{i}-x_{j}} & =\prod_{i \in I ; j \in\{1, \ldots, n\} ; i \neq j} \frac{1-x_{i} x_{j}}{x_{i}-x_{j}} \prod_{i, j \in I ; i \neq j} \frac{1-x_{i} x_{j}}{x_{i}-x_{j}} \\
& =(-1)^{\left(\begin{array}{c}
|I| \\
2
\end{array}\right)} \operatorname{det}\left(\frac{1-x_{i}^{2}}{1-x_{i} x_{j}}\right)_{i, j \in I} \prod_{i \in I ; k \in\{1, \ldots, n\} ; k \neq i} \frac{1-x_{i} x_{k}}{x_{i}-x_{k}} \\
& =(-1)^{\left(\begin{array}{c}
|I| \\
2
\end{array}\right)} \operatorname{det}\left(\frac{1-x_{i}^{2}}{1-x_{i} x_{j}} \prod_{1 \leqslant k \leqslant n ; k \neq i} \frac{1-x_{i} x_{k}}{x_{i}-x_{k}}\right)_{i, j \in I}
\end{aligned}
$$

Hence we have

$$
\begin{aligned}
\operatorname{det} & \left(\beta_{i} \delta_{i, j}+\alpha_{i} \frac{1-x_{i}^{2}}{1-x_{i} x_{j}} \prod_{1 \leqslant k \leqslant n ; k \neq i} \frac{1-x_{i} x_{k}}{x_{i}-x_{k}}\right)_{i, j=1}^{n} \\
= & \sum_{I \sqcup J=\{1, \ldots, n\}} \prod_{j \in J} \beta_{j} \operatorname{det}\left(\alpha_{i} \frac{1-x_{i}^{2}}{x_{i}-x_{j}} \prod_{1 \leqslant k \leqslant n ; k \neq i} \frac{1-x_{i} x_{k}}{x_{i}-x_{k}}\right)_{i, j \in I} \\
= & \sum_{I \sqcup J=\{1, \ldots, n\}}(-1)^{\left(\begin{array}{c}
|I| \\
2
\end{array}\right)} \prod_{i \in I} \alpha_{i} \prod_{j \in J} \beta_{j} \prod_{i \in I ; j \in J} \frac{1-x_{i} x_{j}}{x_{i}-x_{j}} .
\end{aligned}
$$

In the context of the sixth Painlevé equation, we specify the sequence of parameters $c_{k}, d_{k}(k=0,1,2, \ldots)$ as the bilateral shifted factorials

$$
\begin{aligned}
c_{k} & =\prod_{i=0}^{k-1}(c-k+1+2 i)=(c-k+1)(c-k+2) \cdots(c+k-1), \\
d_{k} & =\prod_{i=0}^{k-1}(d-k+1+2 i)=(d-k+1)(d-k+2) \cdots(d+k-1) .
\end{aligned}
$$

for $k=0,1,2, \ldots$. Note that

$$
\begin{aligned}
c_{0}=1, \quad c_{1}=c, \quad c_{2}=(c-1)(c+1), \\
c_{3}=(c-2) c(c+2), \quad c_{4}=(c-3)(c-1)(c+1)(c+3) \ldots .
\end{aligned}
$$

In terms of the ordinary shifted factorials $(c)_{k}=c(c+1) \cdots(c+k-1)$, the bilateral shifted factorials defined above can be expressed as

$$
c_{2 k}=(-1)^{k} 2^{2 k}\left(\frac{1}{2}+c\right)_{k}\left(\frac{1}{2}-c\right)_{k}, \quad c_{2 k+1}=(-1)^{k} 2^{2 k} c(1+c)_{k}(1-c)_{k} .
$$

In this situation, we use the notation $G_{\Lambda}(u, v ; c, d)$ in place of $G_{\Lambda}(u, v ; \boldsymbol{c}, \boldsymbol{d})$. 
In the previous section, we introduced the Umemura polynomials $U_{m, n}(x ; c, d)(m, n \in \mathbb{Z})$ with two discrete parameters as

$$
\begin{aligned}
& U_{m, n}(x ; c, d)=V_{m, n}(x ; a, b) \\
& \text { with } \quad c=2 a+m+n-1, \quad d=2 b-m+n,
\end{aligned}
$$

reparametrizing Masuda's polynomials $V_{m, n}(x ; a, b)(m, n \in \mathbb{Z})$. They have the following symmetries with respect to the discrete parameters $(m, n) \in \mathbb{Z}$ :

$$
\begin{aligned}
& U_{m, n}(x ; c, d)=U_{n, m}(x ; c,-d), \\
& U_{m, n}(x ; c, d)=(-1)^{\left(\begin{array}{c}
m+1 \\
2
\end{array}\right)+\left(\begin{array}{c}
n+1 \\
2
\end{array}\right)} U_{m, n}(x ;-c,-d),
\end{aligned}
$$

and

$$
\begin{aligned}
& U_{m, n}(x ; c, d)=U_{-1-m, n}(x ; c, d) \\
& \quad=U_{m,-1-n}(x ; c, d)=U_{-1-m,-1-n}(x ; c, d) .
\end{aligned}
$$

Note also that

$$
U_{0,0}(x ; c, d)=U_{0,-1}(x ; c, d)=U_{-1,0}(x ; c, d)=U_{-1,-1}(x ; c, d)=1 .
$$

Conjecture 4.5. - Supposing that $n \geqslant m \geqslant 0$, we take the indexing set

$$
\Lambda_{m, n}=\{n+m, n+m-2, \ldots, n-m+2, n-m, n-m-1, \ldots, 1\}
$$

corresponding to the sum $(m, m-1, \ldots, 1)+(n, n-1, \ldots, 1)$ of two staircase partitions. Then we have

$$
U_{m, n}(x ; c, d)=(-1)^{\left(\begin{array}{c}
m+1 \\
2
\end{array}\right)} G_{\Lambda_{m, n}}(u, v ; c, d)
$$

under the identification of variables $u=x+1, v=x-1$.

Note that the original Umemura polynomials that we discussed in Section 1 are the special cases $U_{n-1, n-1}(x ; c, d)=$ const. $U_{n}(x ; c, d)(n \in \mathbb{Z})$. It means that this conjecture has been proved for $U_{n, n}(x ; c, d)(n \in \mathbb{Z})$ by Taneda. We include in Table 4.1 a list of Umemura polynomials $U_{m, n}(x ; c, d)$ in the variables $(u, v)$ with small $m, n \geqslant 0$ with $m \leqslant n$. For small $m, n$, one can verify by computer that $U_{m, n}(x ; c, d)$ coincides up to sign with $G_{\Lambda_{m, n}}(u, v ; c, d)$ defined by the combinatorial formula (4.1).

To conclude this paper, I would like to tell a personal story about Umemura polynomials. As I mentioned already, it was around 1995 that Umemura started to investigate $\tau$ functions associated with the Schlesinger transformations of algebraic solutions appearing as fixed points of Dynkin diagram automorphisms. He was very much excited in recognizing that the recurrence formula (1.1) generates polynomials only. When he brought us $U_{4}$ and $U_{5}$ in the original coordinate in the spring of $1996, U_{4}$ was already a lengthy polynomial of one page, and $U_{5}$ occupied more than five pages. It was a kind of spectacular to see, every time we run the computer, a gigantic 
Table 4.1. Umemura polynomials $U_{m, n}=U_{m, n}(x ; c, d)(u=x+1$, $v=x-1)$

$$
\begin{aligned}
& U_{0,0}=1 \\
& U_{0,1}=c_{1} u+d_{1} v \\
& U_{0,2}=c_{2} c_{1} u^{3}+3 c_{2} d_{1} u^{2} v+3 c_{1} d_{2} u v^{2}+d_{2} d_{1} v^{3} \\
& U_{0,3}=c_{3} c_{2} c_{1} u^{6}+6 c_{3} c_{2} d_{1} u^{5} v+15 c_{3} c_{1} d_{2} u^{4} v^{2}+10 c_{3} d_{2} d_{1} u^{3} v^{3} \\
& +10 c_{2} c_{1} d_{3} u^{3} v^{3}+15 c_{2} d_{3} d_{1} u^{2} v^{4}+6 c_{1} d_{3} d_{2} u v^{5}+d_{3} d_{2} d_{1} v^{6} \\
& U_{0,4}=c_{4} c_{3} c_{2} c_{1} u^{10}+10 c_{4} c_{3} c_{2} d_{1} u^{9} v+45 c_{4} c_{3} c_{1} d_{2} u^{8} v^{2}+50 c_{4} c_{3} d_{2} d_{1} u^{7} v^{3} \\
& +70 c_{4} c_{2} c_{1} d_{3} u^{7} v^{3}+175 c_{4} c_{2} d_{3} d_{1} u^{6} v^{4}+35 c_{3} c_{2} c_{1} d_{4} u^{6} v^{4}+126 c_{4} c_{1} d_{3} d_{2} u^{5} v^{5} \\
& +126 c_{3} c_{2} d_{4} d_{1} u^{5} v^{5}+35 c_{4} d_{3} d_{2} d_{1} u^{4} v^{6}+175 c_{3} c_{1} d_{4} d_{2} u^{4} v^{6}+70 c_{3} d_{4} d_{2} d_{1} u^{3} v^{7} \\
& +50 c_{3} c_{2} d_{1} d_{4} u^{3} v^{7}+45 c_{2} d_{4} d_{3} d_{1} u^{2} v^{8}+10 c_{1} d_{4} d_{3} d_{2} u v^{9}+d_{4} d_{3} d_{2} d_{1} v^{10} \\
& U_{1,1}=c_{2} u^{2}-d_{2} v^{2} \\
& U_{1,2}=c_{3} c_{1} u^{4}+2 c_{3} d_{1} u^{3} v-2 c_{1} d_{3} u v^{3}-d_{3} d_{1} v^{4} \\
& U_{1,3}=c_{4} c_{2} c_{1} u^{7}+5 c_{4} c_{2} d_{1} u^{6} v+9 c_{4} c_{1} d_{2} u^{5} v^{2}+5 c_{4} d_{2} d_{1} u^{4} v^{3} \\
& -5 c_{2} c_{1} d_{4} u^{3} v^{4}-9 c_{2} d_{4} d_{1} u^{2} v^{5}-5 c_{1} d_{4} d_{2} u v^{6}-d_{4} d_{2} d_{1} v^{7} \\
& U_{1,4}=c_{5} c_{3} c_{2} c_{1} u^{11}+9 c_{5} c_{3} c_{2} d_{1} u^{10} v+35 c_{5} c_{3} c_{1} d_{2} u^{9} v^{2}+35 c_{5} c_{3} d_{2} d_{1} u^{8} v^{3} \\
& +40 c_{5} c_{2} c_{1} d_{3} u^{8} v^{3}+90 c_{5} c_{2} d_{3} d_{1} u^{7} v^{4}+56 c_{5} c_{1} d_{3} d_{2} u^{6} v^{5}-14 c_{3} c_{2} c_{1} d_{5} u^{6} v^{5} \\
& +14 c_{5} d_{3} d_{2} d_{1} u^{5} v^{6}-56 c_{3} c_{2} d_{5} d_{1} u^{5} v^{6}-90 c_{3} c_{1} d_{5} d_{2} u^{4} v^{7}-40 c_{3} d_{5} d_{2} d_{1} u^{3} v^{8} \\
& -35 c_{2} c_{1} d_{5} d_{3} u^{3} v^{8}-35 c_{2} d_{5} d_{3} d_{1} u^{2} v^{9}-9 c_{1} d_{5} d_{3} d_{2} u v^{10}-d_{5} d_{3} d_{2} d_{1} v^{11} \\
& U_{2,2}=c_{4} c_{2} u^{6}-3 c_{4} d_{2} u^{4} v^{2}+3 c_{2} d_{4} u^{2} v^{4}-d_{4} d_{2} v^{6} \\
& U_{2,3}=c_{5} c_{3} c_{1} u^{9}+3 c_{5} c_{3} d_{1} u^{8} v-8 c_{5} c_{1} d_{3} u^{6} v^{3}-6 c_{5} d_{3} d_{1} u^{5} v^{4} \\
& +6 c_{3} c_{1} d_{5} u^{4} v^{5}+8 c_{3} d_{5} d_{1} u^{3} v^{6}-3 c_{1} d_{5} d_{3} u v^{8}-d_{5} d_{3} d_{1} v^{9} \\
& U_{2,4}=c_{6} c_{4} c_{2} c_{1} u^{13}+7 c_{6} c_{4} c_{2} d_{1} u^{12} v+18 c_{6} c_{4} c_{1} d_{2} u^{11} v^{2}+14 c_{6} c_{4} d_{2} d_{1} u^{10} v^{3} \\
& -25 c_{6} c_{2} c_{1} d_{4} u^{9} v^{4}-63 c_{6} c_{2} d_{4} d_{1} u^{8} v^{5}-50 c_{6} c_{1} d_{4} d_{2} u^{7} v^{6}+14 c_{4} c_{2} c_{1} d_{6} u^{7} v^{6} \\
& -14 c_{6} d_{4} d_{2} d_{1} u^{6} v^{7}+50 c_{4} c_{2} d_{6} d_{1} u^{6} v^{7}+63 c_{4} c_{1} d_{6} d_{2} u^{5} v^{8}+25 c_{4} d_{6} d_{2} d_{1} u^{4} v^{9} \\
& -14 c_{2} c_{1} d_{6} d_{4} u^{3} v^{10}-18 c_{2} d_{6} d_{4} d_{1} u^{2} v^{11}-7 c_{1} d_{6} d_{4} d_{2} u v^{12}-d_{6} d_{4} d_{2} d_{1} v^{13} \\
& U_{3,3}=c_{6} c_{4} c_{2} u^{12}-6 c_{6} c_{4} d_{2} u^{10} v^{2}+15 c_{6} c_{2} d_{4} u^{8} v^{4}-10 c_{6} d_{4} d_{2} u^{6} v^{6} \\
& -10 c_{4} c_{2} d_{6} u^{6} v^{6}+15 c_{4} d_{6} d_{2} u^{4} v^{8}-6 c_{2} d_{6} d_{4} u^{2} v^{10}+d_{6} d_{4} d_{2} v^{12} \\
& U_{3,4}=c_{7} c_{5} c_{3} c_{1} u^{16}+4 c_{7} c_{5} c_{3} d_{1} u^{15} v-20 c_{7} c_{5} c_{1} d_{3} u^{13} v^{3}-20 c_{7} c_{5} d_{3} d_{1} u^{12} v^{4} \\
& +36 c_{7} c_{3} c_{1} d_{5} u^{11} v^{5}+64 c_{7} c_{3} d_{5} d_{1} u^{10} v^{6}-20 c_{5} c_{3} c_{1} d_{7} u^{9} v^{7}-45 c_{7} c_{1} d_{5} d_{3} u^{8} v^{8} \\
& -45 c_{5} c_{3} d_{7} d_{1} u^{8} v^{8}-20 c_{7} d_{5} d_{3} d_{1} u^{7} v^{9}+64 c_{5} c_{1} d_{7} d_{3} u^{6} v^{10}+36 c_{5} d_{7} d_{3} d_{1} u^{5} v^{11} \\
& -20 c_{3} c_{1} d_{7} d_{5} u^{4} v^{12}-20 c_{3} d_{7} d_{5} d_{3} u^{3} v^{13}+4 c_{1} d_{7} d_{5} d_{3} u v^{15}+d_{7} d_{5} d_{3} d_{1} v^{16} \\
& U_{4,4}=c_{8} c_{6} c_{4} c_{2} u^{20}-10 c_{8} c_{6} c_{4} d_{2} u^{18} v^{2}+45 c_{8} c_{6} c_{2} d_{4} u^{16} v^{4}-50 c_{8} c_{6} d_{4} d_{2} u^{14} v^{6} \\
& -70 c_{8} c_{4} c_{2} d_{6} u^{14} v^{6}+175 c_{8} c_{4} d_{6} d_{2} u^{12} v^{8}+35 c_{6} c_{4} c_{2} d_{8} u^{12} v^{8}-126 c_{8} c_{2} d_{6} d_{4} u^{10} v^{10} \\
& -126 c_{6} c_{4} d_{8} d_{2} u^{10} v^{10}+35 c_{8} d_{6} d_{4} d_{2} u^{8} v^{12}+175 c_{6} c_{2} d_{8} d_{4} u^{8} v^{12}-70 c_{6} d_{8} d_{4} d_{2} u^{6} v^{14} \\
& -50 c_{4} c_{2} d_{8} d_{6} u^{6} v^{14}+45 c_{4} d_{8} d_{6} d_{2} u^{4} v^{16}-10 c_{2} d_{8} d_{6} d_{4} u^{2} v^{18}+d_{8} d_{6} d_{4} d_{2} v^{20}
\end{aligned}
$$


polynomial of the right-hand side divides by another gigantic polynomial. I was completely stunned by these polynomials.

There were two workshops at CRM, Montreal, in two consecutive weeks of May, 1996; one on Painlevé equations and the other on $q$-special functions. It was lucky that Okamoto, Umemura and I had a chance to stay at CRM together in the same period. We discussed many things about Painlevé equations walking around the university. When we started to talk on Umemura's computation, Okamoto suggested: If the sixth Painlevé equation is the counterpart of the hypergeometric equation in the world of nonlinear differential equations, its particular solutions should share the best properties of hypergeometric functions; if it is the case, we know $x= \pm 1$ are the singular points in the case of Umemura's polynomials, and so, the values of $U_{n}$ at these points should factorize! It was a rainy weekend in Montreal, I was troubled by a heavy toothache, but I had to go to work at an office of CRM as I had promised with Okamoto. It was a surprise to both Okamoto and me to see the values of $U_{n}$ at $x= \pm 1$ factorize, but in an unexpected way. We realized that these values are products of quadratic (bilateral) shifted factorials; we were actually seeing the coefficients at the both ends $u^{n(n-1)}$ and $v^{n(n-1)}$ of $U_{n}$. I am not sure whether we already knew that Jacobi polynomials on the interval $[-1,1]$ have nicely factorized coefficients when expressed as a homogenous polynomial of $x+1$ and $x-1$. In any event, Okamoto and I decided to rewrite Umemura's polynomials in the symmetric, homogenous form in terms of $u=x+1$ and $v=x-1$. As I have already shown in Introduction, the results were astonishing. The coefficients of $U_{n+1}$ in the homogenous expansion also seemed to be products of quadratic shifted factorials; they were built up according how to divide the indexing set $\{1, \ldots, n\}$ into two parts. Once we decided to express $U_{n+1}$ as a sum over the pairs $(I, J)$ of subsets with $I \sqcup J=\{1, \ldots, n\}$, it was a fun for us to find the way to the final form of formula (1.9).

Umemura was amazed with these computations of Okamoto and me. The gigantic dragon of $U_{5}$ is now a lovely polynomial of four lines! But, what are the mysterious positive integers $n_{I, J}$ appearing in the coefficients? Umemura would bring these new formulas for $U_{n}$ to anyone who might be interested in combinatorics to ask this question. It was a half year later that we met a third surprise. Towards the end of 1996, we held a workshop on hypergeometric systems at Kobe University. On that occasion, I had a chance to show those new formulas for $U_{n}$ to Okada who was a participant of the workshop. The next morning, Okada came to me and said with all smiles: These are dimensions of irreducible representations of GL. Wow! Again, I was completely knocked out by this. 
This was how Umemura, Okamoto, Okada and I came to write the joint paper "Special polynomials, II". I think that this story of Umemura polynomials was one of the most exciting events in my life. The NOOU conjecture was proven in a couple of years by Taneda, who was a student of Umemura. Referring to it, Umemura wrote in [19]:

This theorem should be counted as a landmark in the history of studies of the Painlevé equations. It clarified the relationship between combinatorics of Young diagrams and the Painlevé equations that cannot be imagined at all in view of the origin of Painlevé equations. At this point in time, I thought we could definitely say that the Painlevé equations define special functions.

To understand the mystery of Umemura polynomials - it was also the main motivation of my later works on Painlevé equations with Yamada and other colleagues. Although more than twenty years have passed since I met Umemura polynomials, I feel there are so many things, or I should say more things than before, that we do not really understand yet about Painlevé equations. The conjecture that I formulated in the present paper is one of the things being left unsolved around myself. I would respectfully pray for Professor Umemura, recollecting irreplaceable experiences with him.

\section{Bibliography}

[1] G. Filipuk, "On the middle convolution and birational symmetries of the sixth Painlevé equation", Kumamoto J. Math. 19 (2006), p. 15-23.

[2] Y. Haraoka \& G. Filipuk, "Middle convolution and deformation for Fuchsian systems", J. Lond. Math. Soc. 76 (2007), p. 438-450.

[3] H. Kageyama, "Painlevé equations and Umemura polynomials", 2003, Master's thesis, Kobe University, in Japanese.

[4] A. N. Kirillov \& M. TANedA, "Generalized Umemura polynomials", Rocky Mt. J. Math. 32 (2002), no. 2, p. 691-702, in Conference on Special Functions (Tempe, AZ, 2000).

[5] - "Generalized Umemura polynomials and the Hirota-Miwa equation", in MathPhys odyssey, 2001, Progress in Mathematics, vol. 23, Birkhäuser, 2002, p. 313331.

[6] O. Lisovyy \& Y. Tykhyy, "Algebraic solutions of the sixth Painlevé equation", J. Geom. Phys. 85 (2014), p. 124-163.

[7] I. G. Macdonald, Symmetric Functions and Hall Polynomials, Oxford Mathematical Monographs, Oxford University Press, 1995.

[8] T. Masuda, "On a class of algebraic solutions to the Painlevé VI equation", Funkc. Ekvacioj 46 (2003), no. 1, p. 121-171.

[9] M. Noumi, Painlevé Equations through Symmetry, Translations of Mathematical Monographs, vol. 223, American Mathematical Society, 2004, x+156 pages. 
[10] M. Noumi, S. OKada, K. OKamoto \& H. Umemura, "Special polynomials associated with the Painlevé equations II", in Integrable Systems and Algebraic Geometry (Kyoto/Kobe 1997), World Scientific, 1997, p. 349-372.

[11] M. Noumi \& Y. YAmadA, "Birational Weyl group action arising from a nilpotent Poisson algebra", in Physics and combinatorics 1999 (Nagoya), World Scientific, 2001, p. 287-319.

[12] — "A new Lax pair for the sixth Painlevé equation associated with $\widehat{\mathfrak{s o}}(8)$ ", in Microlocal Analysis and complex Fourier Analysis, World Scientific, 2002, p. 238-252.

[13] D. P. Novikov, "Integral transformation of solutions of a Fuchs-class equation, that corresponds to the Okamoto transformation of the Painlevé VI equation", Theor. Math. Phys. 146 (2006), no. 3, p. 295-303.

[14] K. Окамото, "Studies on the Painlevé equations I. Sixth Painlevé equation $P_{\mathrm{VI}}$ ", Ann. Mat. Pura Appl. 146 (1987), p. 337-381.

[15] M. TANEDA, "Polynomials associated with an algebraic solution of the sixth Painlevé equation", Jpn. J. Math. 27 (2001), no. 2, p. 257-274.

[16] H. Umemura, "Irreducibility of the first differential equation of Painlevé", in $\mathrm{Al}$ gebraic Geometry and Commutative Algebra, vol. 771, Konokuniya Company Ltd., 1988, p. 771-789.

[17] - "Second proof of the irreducibility of the first differential equation of Painlevé", Nagoya Math. J. 117 (1990), p. 125-171.

[18] - "Painlevé equations and classical functions", Sugaku Expo. 11 (1995), no. 4, p. 77-100, translation of Sügaku 47 (1995), p. 341-359.

[19] - "One hundred years of the Painlevé equations", Sūgaku 51 (1999), p. 395420, in Japanese.

[20] - "Special polynomials associated with the Painlevé equations I", Ann. Fac. Sci. Toulouse, Math. 29 (2020), no. 5, p. 1063-1089, Workshop on the Painlevé equations (Montreal, Canada, 1996).

[21] T. Yoshida, "Determinant formula for Umemura polynomials", 2004, Master's thesis, Kobe University, in Japanese. 